\title{
An evidence accumulation model of perceptual discrimination with naturalistic stimuli
}

\author{
Hector Palada $^{1 *}$, Rachel A. Searston ${ }^{2 *}$, Annabel Persson ${ }^{1}$, Timothy Ballard ${ }^{1 * *}$, \\ Mathew B. Thompson ${ }^{3 * *}$
}

Author Note

Hector Palada, Annabel Persson, Timothy Ballard, School of Psychology, The University of Queensland; Rachel A. Searston, School of Psychology, The University of Adelaide; Matthew B. Thompson, Discipline of Psychology, Murdoch University.

Hector Palada was supported by an Australian Government Research Training Program Scholarship. Timothy Ballard was supported by an ARC Discovery Early Career Researcher Award (DE180101340). Correspondence concerning this article should be addressed to Hector Palada, School of Psychology, The University of Queensland, St Lucia, QLD 4072, Australia. E-mail: hector.palada@uqconnect.edu.au.

Raw data files, experiment code, data analysis script and output are available on the Open Science Framework (https://osf.io/kyp4b/).

*These authors contributed equally to this work.

**Shared senior authorship. 


\begin{abstract}
Evidence accumulation models have been used to describe the cognitive processes underlying performance in tasks involving two-choice decisions about unidimensional stimuli, such as motion or orientation. Given the multidimensionality of natural stimuli, however, we might expect qualitatively different patterns of evidence accumulation in more applied perceptual tasks. One domain that relies heavily on human decisions about complex natural stimuli is fingerprint discrimination. We know little about the ability of evidence accumulation models to account for the dynamic decision process of a fingerprint examiner resolving if two different prints belong to the same finger or not. Here, we apply a dynamic decision-making model — the linear ballistic accumulator (LBA) — to fingerprint discrimination decisions in order to gain insight into the cognitive processes underlying these complex perceptual judgments. Across three experiments, we show that the LBA provides an accurate description of the fingerprint discrimination decision process with manipulations in visual noise, speedaccuracy emphasis, and training. Our results demonstrate that the LBA is a promising model for furthering our understanding of applied decision-making with naturally varying visual stimuli.
\end{abstract}

Keywords: Evidence accumulation, linear ballistic accumulator decision models, fingerprint discrimination, perceptual expertise. 
Evidence accumulation models provide a detailed description of the processes underlying rapid human decision making (Donkin \& Brown, 2018). The models assume that a decisionmaker samples evidence from the environment until a threshold amount of evidence is accumulated, at which point an overt response is triggered. Unlike 'static' decision models, such as signal detection (Green \& Swets, 1966), evidence accumulation models account for choice probability and response times, as well as the interaction between the two. The appeal of an evidence accumulation approach to modelling decisions is that it can reveal novel facets of the decision process beyond raw response time, accuracy, and other static measures of performance. While these models have traditionally been applied to two-choice discrimination tasks where the stimuli vary artificially along a single dimension, such as motion or orientation detection tasks (for a review see Ratcliff \& McKoon, 2008), their success has generated interest in potential applications beyond the lab. For instance, how these models fare with decisions about highly variable, multidimensional or noisy stimuli encountered in applied areas such as air traffic control, medical diagnosis or forensic science is unknown.

Recent efforts have shown that accumulation models can account for behavior amidst additional task complexity. For example, this class of model has been applied to air traffic control conflict detection tasks (Loft, Bolland, Humphreys, \& Neal, 2009; Vuckovic, Kwantes, Humphreys, \& Neal, 2014), unmanned aerial vehicle simulation target detection tasks (Palada, Neal, Vuckovic, Martin, Samuels, \& Heathcote, 2016), and medical image decision-making tasks (Trueblood et al., 2018). Using naturally varying stimuli (e.g., medical images) comprising covarying visual features, or more controlled stimuli (e.g., simulated targets) involving simpler decision rules, these prior studies have probed the generality of several different evidence accumulation models. Extending these models even further afield will help to reveal how closely each parameter tracks decision processes with different kinds of naturally varying stimuli, tasks, and participant samples. 
Here we apply the linear ballistic accumulator model (LBA; Brown \& Heathcote, 2008) to a fingerprint discrimination task where participants are faced with accumulated evidence about naturalistic stimuli that bear a strong resemblance to forensic fingerprint evidence encountered in the field. No such model has been used to describe how people arrive at a decision about the identity or source of forensic specimens - fingerprints, handwriting, firearms or hair - despite the analogous "evidence" accumulation nature of such forensic investigative tasks. Across three experiments, we examine whether the LBA can provide a coherent account of decision-making in a task that closely resembles the process of deciding whether a crime scene print matches a suspect ("same" fingerprints) or not ("different" fingerprints). Our first experiment examines the ability of the LBA to account for patterns of choices and response times in a fingerprint discrimination task. Our second experiment examines the effects of standard manipulations such as speed-accuracy emphasis and stimulus difficulty to test whether the model parameters are sensitive to factors they should be, according to theory. Our third experiment examines how a training intervention affects the cognitive processes underlying finger discrimination, as reflected by the model parameters.

In the following sections, we review previous literature on fingerprint discrimination, describe how fingerprints could benefit from evidence accumulation modelling, and illustrate the application of the LBA to fingerprints.

\section{Fingerprint Discrimination}

Fingerprint discrimination is done by humans, not computers. When a fingerprint is found at a crime scene a human examiner-often a police officer — compares the print to a known suspect or to a list of candidate prints. They position two prints side-by-side, physically or on a computer screen, and visually compare them to judge whether they came from the same finger or two different fingers. Compared to novices, these examiners are remarkably accurate at discriminating prints (Tangen, Thompson \& McCarthy, 2011), particularly when they are highly similar in the eyes of a computer algorithm (Thompson, Tangen \& McCarthy, 2013b). 
Experts tend to show a conservative response bias in these experiments, such that they err on the side of making more errors that could allow a guilty person to escape detection than errors that could falsely incriminate an innocent person (Thompson et al., 2013a).

Other experiments on the nature of fingerprint expertise have revealed that fingerprint experts can make quick and relatively accurate decisions in noise and under time pressure compared to novices (Thompson \& Tangen, 2014). They can also maintain this expertise across different fingerprint tasks that bear less of a resemblance to their daily work. For example, they can tell if two prints were left by different fingers of the same individual more accurately than novices (Searston \& Tangen, 2017a), and they show even greater expertise in distinguishing common fingerprint patterns such as "loops" and "whorls" in a search task (Searston \& Tangen, 2017b). However, their expertise also appears to be constrained by their specific set of experiences. For example, a longitudinal investigation of the development of fingerprint expertise revealed that examiners' performance on a range of fingerprints tasks improved as they accumulated 12 months of formal training with fingerprints, but their performance on a series of analogous tasks with inverted face stimuli did not improve (Searston \& Tangen, 2017c).

Signal detection models have been the predominant class of model used to understand decisions in these fingerprint discrimination experiments (Thompson et al., 2013a; Searston, Tangen \& Eva, 2016). These models have allowed researchers to examine factors that influence decision parameters such as response bias (i.e., whether people are biased toward responding "same" vs. "different") and discriminability (i.e., the ability of the decision maker to discriminate same fingers vs. different fingers) separately. For instance, trainees with five weeks to six months experience can be more conservative in their responding (i.e., tended to say "different" more frequently) than novices, who displayed a liberal bias when comparing fingerprints (i.e., tended to say "same" more on the same task; Thompson et al., 2013b). Fingerprint experts demonstrate superior discriminability compared to novices irrespective of 
their response bias (Searston \& Tangen 2017a; Searston \& Tangen, 2017b). These models have also been used to show how contextual information (e.g., case reports of crimes that vary in severity) can influence response bias without necessarily reducing the discriminability of prints from the same individual (Searston, Tangen \& Eva, 2016).

The application of signal detection theory to fingerprint discrimination decisions has provided insights into how experts differ from novices, how context can sway people's decisions, and how people's discriminability can vary with experience. Despite the value of this model for helping to understand fingerprint discrimination, there are aspects of the underlying decision process that remain obscured. That is, signal detection models are static models that do not make use of the response time distribution and so they do not account for the dynamics of the decision process. An increase in hits together with a decrease in false alarms, for example, is typically interpreted as an increase in discriminability. This pattern of results, however, could also be caused by increases in the response threshold that determines when the evidence for a response alternative is sufficient to trigger an overt response (e.g., Rae, Heathcote, Donkin, Lee, \& Brown, 2014). These two processes can be disentangled using evidence accumulation models, which consider both response times and accuracy.

\section{Evidence Accumulation Models}

The term 'evidence accumulation model' refers to a class of cognitive model that describes the process underlying rapid human decision making. These models share the basic assumption that the decision maker accumulates evidence for response alternatives until a threshold amount of evidence is reached, at which point an overt response is triggered. Although modern evidence accumulation models often yield similar conclusions about the decision process regardless of which model is considered, the models make different assumptions about the underlying architecture (Donkin, Brown, Heathcote, \& Wagenmakers, 2011). 
Evidence accumulation models have developed over the last 50 years with architectural extensions added over time to account for more complex empirical observations (see Donkin \& Brown, 2018 for a more detailed review). Early models assumed that a single accumulator indexed the difference in evidence for each choice followed a random walk process; response time variability was captured by the moment-to-moment change in evidence (Stone, 1960). Subsequent models used a continuous accumulation process, or a diffusion process, and added trial-to-trial variability in the evidence accumulation start point and the mean rate of evidence accumulation. These added sources of variability captured differences in response times for correct and error response times (Laming 1968; Ratcliff, 1968). The current diffusion model has also added trial-to-trial variability in non-decision processes to account for variability in the fastest response times across conditions (Ratcliff \& McKoon, 2008). Multiple accumulator models have also been proposed, which assume that evidence accumulates independently for each response alternative(s). The leaky accumulator model (Usher \& McClelland, 2001) assumed response competition between accumulators and within-trial randomness. The latter assumption was subsequently omitted in the simplified ballistic accumulator model (BA; Brown \& Heathcote, 2005). The linear ballistic accumulator (LBA; Brown \& Heathcote, 2008) is a further simplification of the BA model, as it assumes linear evidence accumulation (Brown \& Heathcote, 2005).

The success of evidence accumulation models stems from their ability to provide insights into the latent cognitive processes that underlie choice. The models are typically applied to tasks using simple stimuli that produce rapid decision times $(<1.5 \mathrm{~s})$, such as memory recognition ("was it recently encountered?"), motion discrimination ("is it moving to the left or right?") and lexical decisions (“is it a word or non-word?"). The pairing of evidence accumulation models with these types of tasks has facilitated our understanding of a range of issues, including sleep deprivation (Ratcliff \& Van Dongen, 2011), schizophrenia (Heathcote et al., 2015), and anxiety (Ho et al., 2014). 
There is recent evidence that the LBA can be used to account for decisions in tasks that are inspired by applied domains and produce extended mean response times $(\sim 2.5 \mathrm{~s}$; e.g., Palada et al., 2018). For example, Strickland et al. (2019) and Boag et al. (2019) used the LBA to understand the processes underlying prospective memory in a maritime surveillance task and air traffic control task, respectively. The LBA has also been used to understand how individuals adapt to time pressure in an applied multi-stimulus environment (Palada et al., 2018), and in a dual-task environment with multiattribute stimuli (Palada et al., 2016; Palada, Neal, Strayer, Ballard \& Heathcote, 2019). However, these studies used artificial or controlled stimuli which were quite removed from their inspirations in the field. That is, these studies opted for a high level of control to ensure that the tasks were amenable to evidence accumulation models. For example, the maritime surveillance task used by Strickland et al. (2019) used basic target features and classification rules, and the air traffic control task used in Boag et al. (2019) only presented one pair aircraft pair per trial. In this paper, we examine whether the LBA can provide a coherent account of decisions in the domain of fingerprint discrimination using a task which bears a strong resemblance to the task in the field where the stimuli are far less controlled.

The LBA model architecture is shown in Figure 1. In fingerprint discrimination, a person must decide whether pairs of fingerprints come from the same finger or from different fingers. In this case, the model includes two evidence accumulators, each corresponding to a different response alternative ("same" finger vs. "different" finger). The LBA assumes that the level of evidence in each accumulator at the start of the decision process is sampled from a uniform distribution with a lower boundary of zero and an upper boundary of $A$. The rate of evidence accumulation within a trial is sampled from a normal distribution with mean $v$ and standard deviation $s v$. The rate of evidence accumulation for each accumulator depends on the stimulus. For example, in cases where the two fingerprints come from the same finger, the mean rate of evidence accumulation will be higher for the accumulator corresponding to the 
"same" finger response than for the accumulator corresponding to the "different" finger response. All else being equal, this means that the "same" finger response is more likely to be triggered. A response is triggered when level of evidence in an accumulator breaches the threshold $(b)$ associated with that response alternative. As the two accumulators race towards their respective response threshold, the winning accumulator will make the corresponding response (i.e., a "same" finger response in Figure 1). The response threshold may be the same between the different alternatives (as in Figure 1), or it may differ. Finally, the model includes a non-decision time parameter $\left(T_{e r}\right)$. Observed response times are accounted for by first partitioning the response times into non-decision time and decision time components. Nondecision time reflects the sum of times for stimulus encoding and response production. The decision time, and observed responses, are reflected by the inputs and operation of the evidence accumulation model; hence, the two evidence accumulators racing towards the threshold.

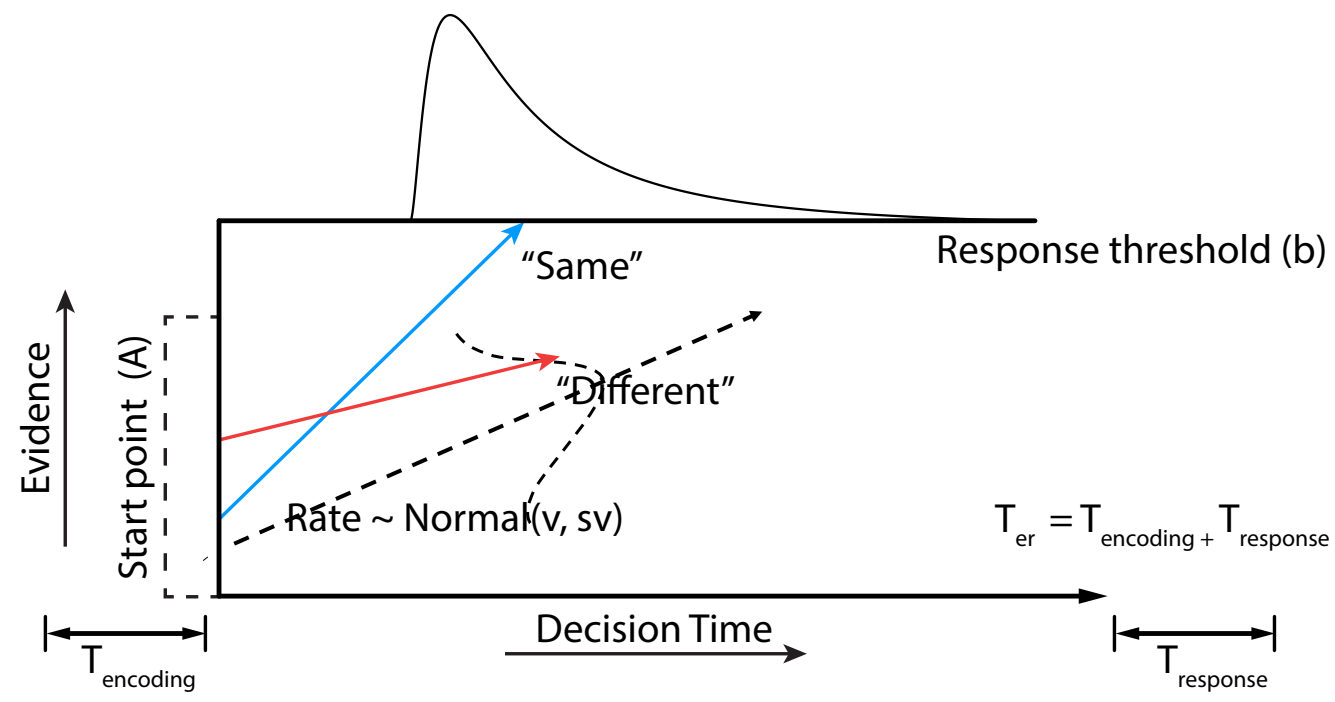

Figure 1. The standard Linear Ballistic Accumulator model applied to fingerprint identification and associated parameters: Response threshold (b), mean rate (v), rate variability (sv), start-point (A), and non-decision time (Ter).

The LBA can be used to quantify the latent cognitive processes underlying decision making. The model captures response caution (i.e., the amount of information required to 
reach a decision) and response bias via the response threshold parameter. Lower response thresholds produce quicker, more error prone responses, because less evidence is feeding into the final decision. Higher response thresholds produce slower, but more accurate responses, because more evidence is being considered. Differences in thresholds between response alternatives produce a bias in favor of the response with the lower threshold, because less evidence is required to trigger that response than the competing response(s). The LBA captures the discriminability of the stimulus via the rate parameters. The mean rate between the two evidence accumulators can be used to account for changes in the speed of information processing and the rate variability parameters captures the heterogeneity in the quality of the stimulus and attention (Palada et al., 2018; Ratcliff, 1978). 


\section{Modeling Fingerprint Discrimination}

We use three experiments to test the ability of the LBA to account for fingerprint discrimination decisions made by novices. Experiment 1 provides an initial test of whether the LBA is able to account for the patterns of choices and response times using an experiment in which the only manipulation is whether the stimuli were from the same finger versus different fingers. Experiment 1, to our knowledge, provides the first application of evidence accumulation models to fingerprint discrimination. Experiment 2 examines whether the parameters of the LBA are sensitive to manipulations of speed-accuracy emphasis and stimulus noise. We use these traditional benchmark manipulations to test whether the parameters of the model can be interpreted in a meaningful way, consistent with the interpretations made in studies involving other types of decisions (Donkin \& Brown, 2018). Experiment 3 uses the LBA to examine the cognitive processes underlying the effects of training on fingerprint discrimination.

\section{Experiment 1}

In Experiment 1, we take the first step in examining how the LBA can be used to understand fingerprint discrimination decisions. Specifically, we test whether the model can account for the empirical patterns of choices and response times when individuals are faced with the task of discriminating pairs of fingerprints from the same finger versus different fingers.

\section{Method}

\section{Participants \& Stimuli}

Thirty-six psychology undergraduate students (28 female and 8 male; mean age $=20.19$ years, $\mathrm{SD}=4.61$ ) from The University of Queensland participated in this experiment for course credit. The experiment was approved by the University of Queensland Human Research Ethics Committee. The stimuli included 195 fingerprint trios, each consisting of a simulated crime scene print, a fully-rolled print from the same finger, and a fully-rolled print 
from a different finger. The "different" prints were sampled randomly for each participant from a pool of 195 prints collected from different individuals. The images were sourced from the Forensic Informatics Biometric Repository (Thompson et al., 2013a), and we cropped them to $512 \times 512$ pixels, isolating the print in the center of the image. Ninety-six fingerprint pairs (48 same-finger prints and 48 different-finger prints) were generated for each participant. A random sample of 48 crime-scene prints, sampled from the pool of 195, were paired with their corresponding "same" print, and a separate set of 48 crime-scene prints, sampled randomly from the remaining pool of 147, were paired with their corresponding "different" print.

\section{Procedure}

After reading an information sheet about the experiment and watching an instructional video with an example same-finger and different-finger trial, we presented participants with 96 fingerprint pairs, one pair at a time. Participants were instructed to judge whether the two prints belong to the same finger, or two different fingers. They provided their judgments by pressing the "Z" or "/" key. On pressing one of the two response keys or after 10 seconds, the two prints disappeared, with a 1 second interval before the next pair were displayed. Participants were encouraged, in the instructional video, to respond within 10 seconds; not doing so recorded an "NA" response. We collected participants' keypresses and time to respond on each trial.

We randomly varied whether a " $Z$ ” keypress indicated a match (i.e., same-finger prints) or mismatch response (i.e., different-finger prints). A random selection of participants were instructed to respond by pressing " $Z$ " if they thought the two prints were from the same finger (and "/" if they thought they were from different fingers), and the remaining participants responded by pressing "/" if they thought the two prints were from the same finger (and " $Z$ " if they thought they were from different fingers). Each participant's particular response-key arrangement was reflected in the instructional video they viewed at the beginning of the 
experiment. We also displayed a $1376 \times 848$ image of the keyboard at the bottom center of the computer screen throughout the experiment with the labels "Same" and "Different" in bold, black text above the two corresponding response keys. The keyboard image was semitransparent, other than the two response keys and the labels (see Figure 2 for a screenshot of what participants' see on a given trial).

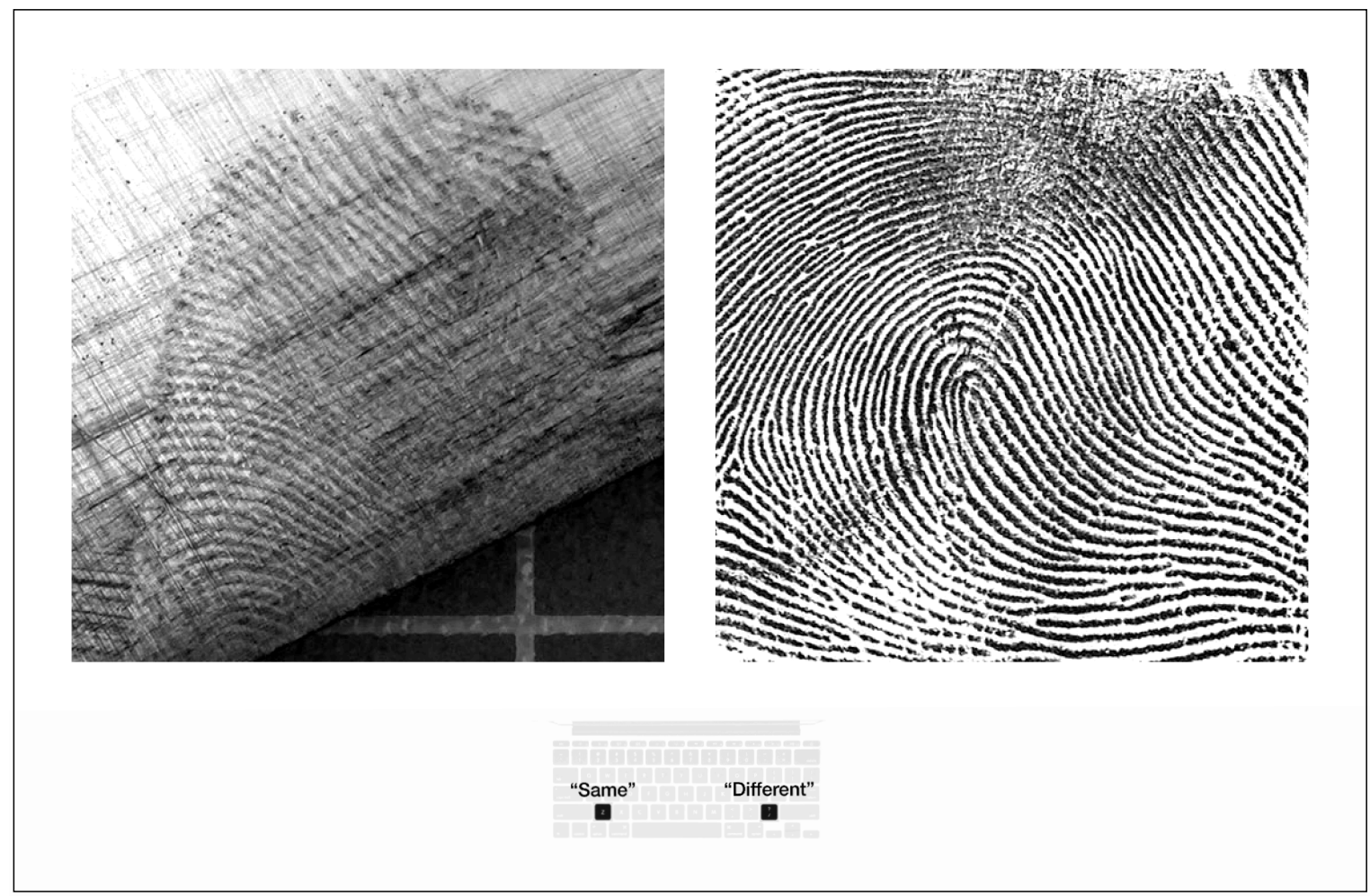

Figure 2. A screenshot of a single trial. Participants in all three experiments were presented with the same basic visual display of two fingerprints (same-finger prints in this instance) and a keyboard response map on each trial.

\section{Results}

Censoring of rapid RTs was not required as the fastest observed RT (311ms) was sufficient to make a valid decision. Extended RTs $(<10 \mathrm{~s})$ were not censored so that we could account for the entire distribution of RT performance. We removed three participants with a high nonresponse rates $(>10 \%)$, where one participant had a $20 \%$ non-response rate, and two participants both had a $27 \%$ non-response rate. The remaining participants had $10 \%$ or less missing data. 


\section{Discrimination Performance}

We examined the effect of the stimulus type manipulation on the RTs for correct responses, the RTs for incorrect responses, and accuracy. These analyses were run using R ( $R$ Development Core Team, 2016), using the brms package to conduct Bayesian generalized linear mixed-models (GLMM; Bürker, 2017). Priors were consistent across outcomes variables (Cauchy prior; location $=0$, scale $=2$ ) for fixed effects and random intercepts; the latter was zero-truncated. Other priors used the default specifications in brms. We present the posterior estimates of regression coefficients $(b)$ with two-tailed $95 \%$ credible intervals (CIs) presented in square brackets. We infer credible effects where the CI does not cross zero (Kruschke, 2014).

Correct and incorrect RTs quantiles $(0.1,0.5$, and 0.9$)$, and mean accuracy are shown in Figure 3. Stimulus type did not have a credible effect on correct RTs $(b=-0.02[-0.04,0.00]$; same-finger $=3.15 \mathrm{~s}$ vs. different-finger $3.27 \mathrm{~s})$ or on incorrect RTs $(b=0.03[0.00,0.06]$; $3.64 \mathrm{~s}$ vs. $3.43 \mathrm{~s})$. There was a credible effect of stimulus type on accuracy, such that participants were less accurate when responding to same-finger prints compared to differentfinger prints $(b=-0.11[-0.19,-0.04] ; 67 \%$ vs. $71 \%)$.

\section{LBA Modeling and Analysis}

To apply the LBA, the researcher must specify which model parameters should be allowed to vary with experimental factors and accumulator related factors. Table 1 outlines the model parameterizations and the total number of parameters for the three experiments. For Experiment 1, mean rate $(v)$ is allowed to vary depending on the stimulus factor ("same" finger vs. "different" fingers). We allow mean rate to vary with a "match" factor, which reflects whether the response that the accumulator triggers "matched" the stimulus presented in the trial. In other words, the match factor indicates whether the accumulator corresponded to the correct response for the presented stimulus. The accumulator for the correct response is referred to as the "matching" accumulator, because the response it triggers "matches" the 
stimulus. The accumulator for the incorrect response is referred to as the "mismatching" accumulator, because the response it triggers "mismatches" the stimulus. We allow rate variability $(s v)$ to vary by the match and stimulus factors, with the latter hypothesized to account for heterogeneity in the features between same prints and different prints. We allow threshold (b) to vary by the response factor ("same" finger vs. "different" fingers) so that we can account for any biases in responding. As is common practice (e.g., Brown \& Heathcote, 2008), we express response caution as the difference between the raw threshold and the maximum starting point of evidence (denoted $B)$. This provides a pure measure of caution that is not contaminated by individual differences in starting point variability. Starting point variability $(A)$ and non-decision time $(t 0)$ are constrained to a single estimated value. To ensure that the model is identifiable, we constrain the rate variability for the mismatching accumulator of the "different" finger pairs to one (Donkin, Brown, \& Heathcote, 2009). 
Table 1.

Linear ballistic accumulator model parameterization for the three experiments.

\begin{tabular}{llcccccc}
\hline Experiment & Model & $\begin{array}{c}\text { Start } \\
\text { point } \\
(\mathrm{A})\end{array}$ & $\begin{array}{c}\text { Threshold } \\
(\mathrm{B})\end{array}$ & $\begin{array}{c}\text { Rate } \\
(\mathrm{v})\end{array}$ & $\begin{array}{c}\text { Rate } \\
\text { variability } \\
\left(s_{\mathrm{v}}\right)\end{array}$ & $\begin{array}{c}\text { Non- } \\
\text { decision } \\
\text { time } \\
\left(\mathrm{t}_{\mathrm{er}}\right)\end{array}$ & Total \\
\hline Experiment 1 & Factors & - & $\mathrm{R}$ & $\mathrm{S}, \mathrm{M}$ & $\mathrm{S}, \mathrm{M}$ & - & 11 \\
& $\mathrm{NF}$ & 1 & 2 & 4 & 3 & 1 & 25 \\
\hline Experiment 2 & Factors & - & $\mathrm{SA}, \mathrm{R}$ & $\mathrm{SA}, \mathrm{N}, \mathrm{M}, \mathrm{S}$ & $\mathrm{S}, \mathrm{M}$ & - & \\
& $\mathrm{NF}$ & 1 & 4 & 16 & 3 & 1 & 21 \\
\hline & Factors & - & $\mathrm{F}, \mathrm{B}, \mathrm{R}$ & $\mathrm{F}, \mathrm{B}, \mathrm{M}, \mathrm{S}$ & $\mathrm{F}, \mathrm{B}, \mathrm{S}, \mathrm{M}$ & - & \\
\hline Experiment 3 & $\mathrm{NF}$ & 1 & 4 & 8 & 7 & 1 & 2 \\
\hline
\end{tabular}

Note. Experimental factors are stimulus type (S), speed-accuracy emphasis (SA), noise (N), feedback (F) and block (B). Accumulator factors are match (M) and response (R). "Total" refers the total number of parameters in the model; NF = number of factors. Note: In Experiment 3, feedback was manipulated between-person; we fit the two feedback groups separately, therefore feedback factor $(\mathrm{F})$ does not contribute to the number of model parameters.

We implemented the LBA using a hierarchical Bayesian framework, which assumes that parameters vary across individuals but are drawn from common population distributions. The individual-level parameters were modeled using normal or truncated normal distributions. The $A, B$, and $s v$, parameters had a lower bound of 0 and no upper bound. The $t 0$ parameter had a lower bound of 0.1 and an upper bound of 1 . The $v$ parameters were unbounded. The population distribution for each individual-level parameter requires two population-level parameters: location and scale. The priors of the location and scale parameters were modeled using normal or truncated normal distributions. The details of the priors are provided in supplementary materials. The posterior distributions were estimated using the differential evolution MCMC algorithm (Turner, Sederberg, Brown, \& Steyvers, 2013), as implemented by the Dynamic Models of Choice package in R (Heathcote, Lin, Reynolds, Strickland, Gretton, Matzke, 2018; see supplementary materials for information about the number of chains and assessing convergence). 
As can be seen in Figure 3, the model provides a good fit to mean accuracy for both types of stimuli. The model also provides a good fit to the RT distribution for correct and incorrect responses. There is some evidence of underestimation of RTs for the 0.9 quantile, with data falling just outside of the credible interval of the model predictions. However, such overestimation at the higher quantiles is common when modeling complex tasks (e.g., Palada et al., 2016 \& Palada et al., 2018).
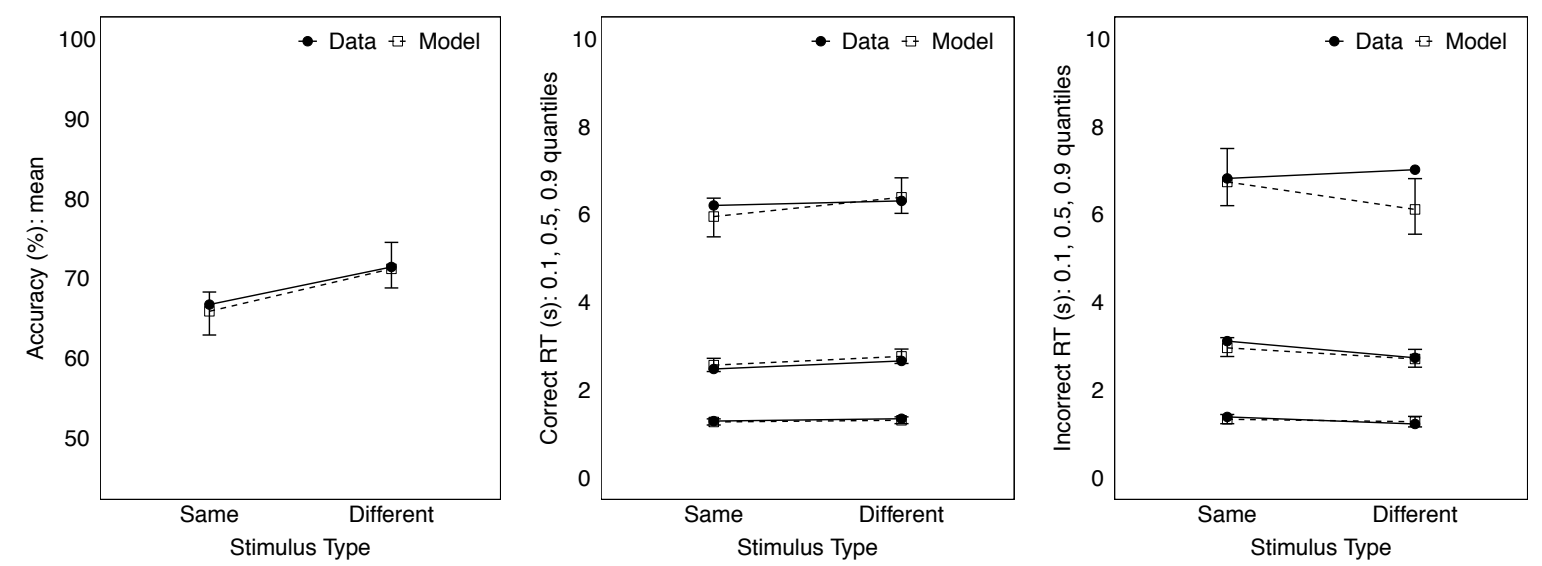

Figure 3. Experiment 1: Fits of the LBA to mean accuracy (left graph), and quantiles of correct (middle graph) and incorrect response times (right graph) of the fingerprint discrimination task. The bars show the 95\% quantiles of the posterior predictives.

We next examined the posterior estimates of model parameters to examine how they accounted for the patterns observed in performance data, which are illustrated in Figure 4. To make inferences about parameters, and account for the uncertainty in posteriors, we create group-averaged posterior distributions by averaging every posterior sample across participants. To test for differences between parameters, we calculated the difference between parameters for each condition, posterior sample, and participant, and then averaged over participants to produce a group average posterior distribution on the difference in parameters. To test for an interaction between two factors with two levels each, we calculated the difference between levels for one factor for each of the two levels for the other factor, thus producing two contrasts. We then calculated the difference between the two contrasts (Heathcote et al., 2018; Palada et al., 2019). 
There was a credible difference in the threshold for the two response types, $0.25[0.14$, 0.37], with participants setting a lower threshold for the "different" response than the "same" response. The differences between mean rate for the matching and mismatching accumulators was credibly greater for different-finger than same-finger print pairs, $1.30[1.10,1.51]$. There was a credible interaction between stimulus type and match factor on variability in the rate of evidence accumulation, $-0.99[-1.13,-0.84]$, such that for same-finger prints, the rate variability for the matching (i.e., correct) accumulator was greater than the rate variability for the mismatching (i.e., incorrect) accumulator, whereas the opposite occurred for differentfinger prints, though to a lesser extent. In the LBA, greater rate variability for the matching accumulator can account for the typically observed slow error response times. In contrast, greater variability in the mismatching accumulator compared to the matching accumulator can account for relatively fast errors (Heathcote \& Love, 2012), and as shown in Figure 3, errors were relatively faster for different-finger pairs compared to same-finger pairs.

Under the LBA architecture, greater evidence quality can be driven by a greater difference between mean rates between the two accumulators, differences in rate variability, or both. Figure 5 plots the rate of evidence accumulation using the median of the posterior estimates of mean rates and rate variability. The distributions illustrate the analogues of signal and noise distributions from SDT, and therefore allows for a inferences of the analogues of sensitivity (i.e., $d$ '), as well as the causes of any differences in sensitivity. The graph illustrates how sensitivity was greater for different-finger pairs compared to same-finger pairs due to the greater differences in means, and the lesser variability for the matching accumulator, though this was slightly offset by having greater variability for the mismatching accumulator.

Overall, the greater accuracy for different-finger compared to same-finger pairs was driven by the greater difference between the two accumulators, as well as the bias to respond "different". While the bias to respond "different" would typically result in faster response 
times for different-finger prints than same-finger prints, as the accumulator has a shorter distance to reach the threshold, this appeared to be offset by two separate factors. First, in the case of the correct response times, the matching (i.e., correct) accumulator of the differentfinger pairs had a less variable rate than the accumulator of the same-finger pairs. Secondly, in the case of error response times, the mismatching (i.e., incorrect) accumulator of the different-finger pairs had a mean rate that was lower than the accumulator of the same-finger pairs.
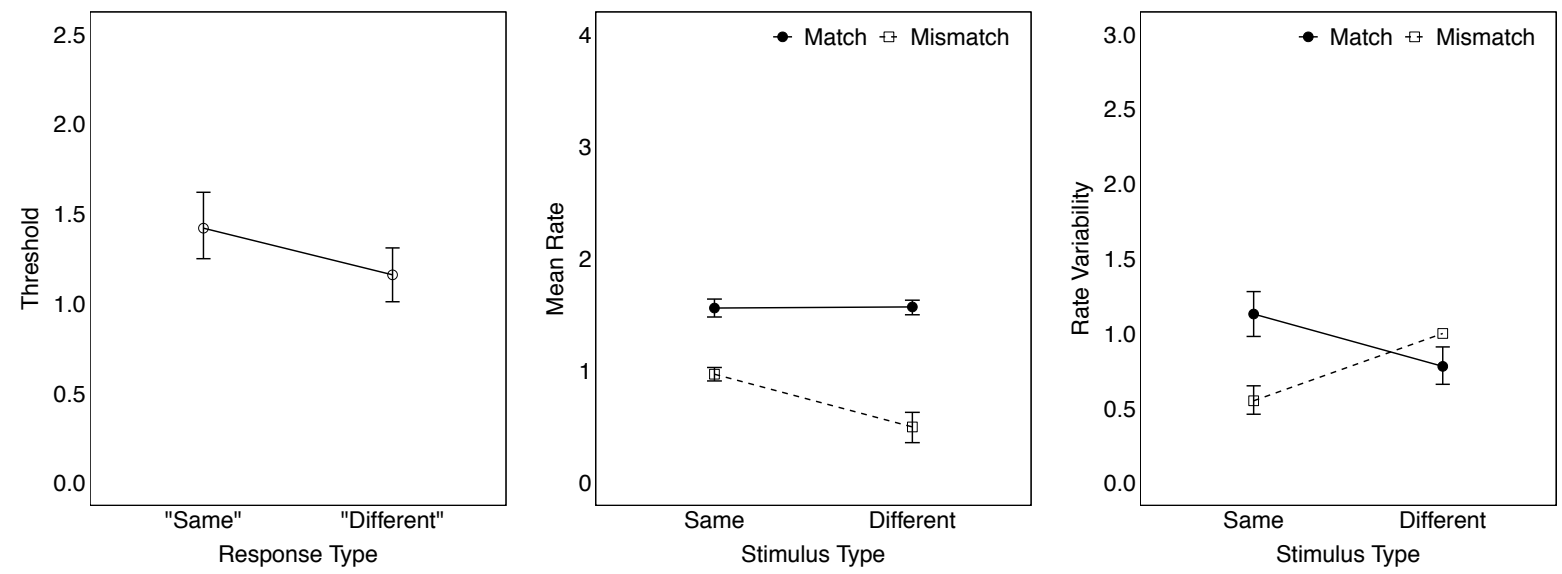

Figure 4. Experiment 1: Median parameters estimates for the LBA model, including response caution (left panel), mean rate (middle panel) and rate variability (right panel). The bars show the 95\% credible intervals.

Match (i.e., correct) $\square$ Mismatch (i.e., incorrect)

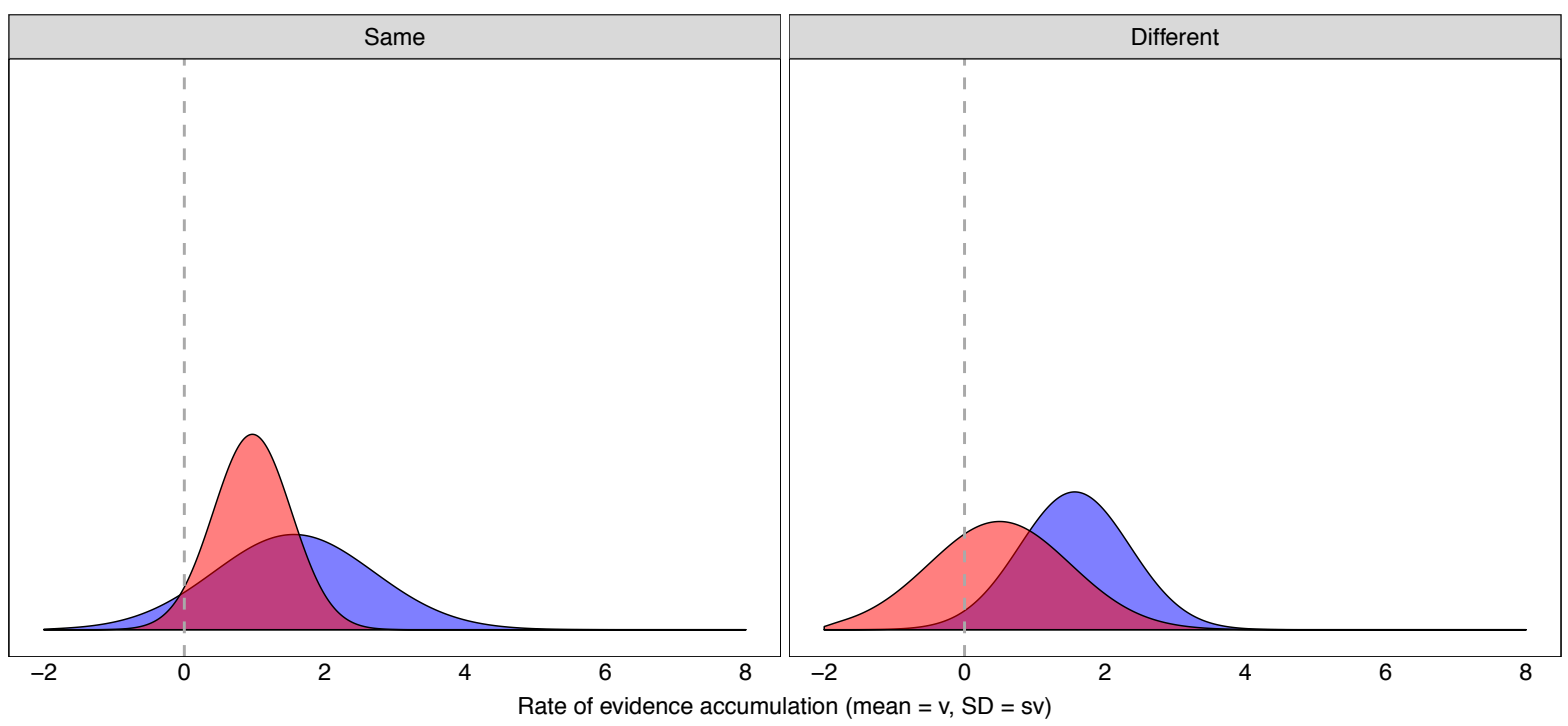


Figure 5. Experiment 1: Distribution plots of rates of evidence accumulation for matching and mismatching accumulators. Columns refer to stimulus type. Distributions were generated using posterior medians. Vertical line reflects zero truncation of rate sampling.

\section{Discussion}

To our knowledge, this experiment was the first application of the LBA to examine performance observed in a fingerprint discrimination task. We found that the model provided a good fit of the observed accuracy and full distribution of correct and incorrect RTs. Moreover, the LBA revealed aspects of the decision-making processes that could not be inferred from the performance data alone.

Participants required more evidence before deciding that prints were left by the same finger, compared to deciding they were left by different fingers, indicating that participants were biased towards responding "different". The bias to respond "different" is contrary to previous studies applying signal detection theory to fingerprint discrimination (Thompson et al., 2013a; Searston, Tangen, \& Eva, 2015). However, this prior research used highly similar different-finger pairs that were intended to be challenging for both novices and expert. We did not use highly similar different-finger pairs. Rather, we used dissimilar different-finger pairs by randomly pairing fingerprints so that we would have a sufficient number of different-finger trials for model fitting. As a result, the different-finger pairs appeared more different than pairs used in previous research. We also used simulated crime scene prints which result in considerable variability among same-finger pairs due to distortion, pressure, partial contact and surface type. Therefore, our same-finger pairs tended to appear different due to the different features that were visible. These features of our design together may have contributed to the "different" response being more salient in our paradigm.

The model revealed that participants had greater discriminability for different-finger pairs than same-finger pairs. The model also revealed greater variability in the rate of evidence accumulation of the matching accumulator for same-finger pairs compared to 
different-finger pairs, which suggests that the processing of same-finger pairs was more heterogenous than different-finger pairs. Our use of dissimilar different-finger pairs and crime-scene prints may explain why we observed these effects. Specifically, identifying different-finger pairs can be done effectively and with little variability because the individual could easily identify dissimilar pairs of fingerprints. In contrast, identifying same-finger pairs could require additional and more variable processing because the individual needs to conduct an exhaustive evaluation of the within-finger differences in order to rule them out as merely contextual differences that resulted from them being crime-scene prints.

\section{Experiment 2}

Experiment 1 provided initial evidence that the LBA can account for performance in a fingerprint discrimination task. However, adequate model fit to observed data is insufficient to establish model validity. To build stronger evidence that the LBA can be used to understand fingerprint discrimination, we next examine whether the model parameters change in predictable ways in response to experimental manipulations and our theoretical understanding of their effects (Donkin \& Brown, 2018). In Experiment 2, we examine the interpretability of model parameters by manipulating emphasis type (speed vs. accuracy) and noise (no noise vs. noise). We expect that emphasis type will affect parameters associated with the termination of the decision process itself (i.e., response threshold), whereas noise will affect parameters associated with the inputs to the decision process (i.e., rate of evidence accumulation). However, as we describe in the LBA modeling and analysis section, and consistent with current modeling procedures, we test for the possibility that emphasis type also affects the rate of evidence accumulation (Rae et al. 2014; Palada et al., 2016).

\section{Method}

\section{Participants \& Stimuli}

A second group of 70 psychology undergraduates (36 female, 34 male) with an average age of $19.71(\mathrm{SD}=5.01)$ from The University of Queensland participated in Experiment 2 in return 
for course credit. The image-set was the same used in Experiment 1. We duplicated this entire set of images and added $20 \%$ of artificial noise or 'speckle' to the duplicates, such that there was a noise and no noise 'twin' of every image in the set (see Figure 6). For each participant, we then randomly sampled a total of 192 fingerprint trios, with a random half of these (96 trios) taken from the no noise set, and the other half taken from the duplicated noisy set. That is, the fingerprints were randomly and equally sampled from the noise or no noise sets for each participant. For each of the noise and no noise image sets, a random 48 crime-scene prints were paired with their corresponding same-finger print, and 48 crime-scene were paired with their corresponding different-finger print.
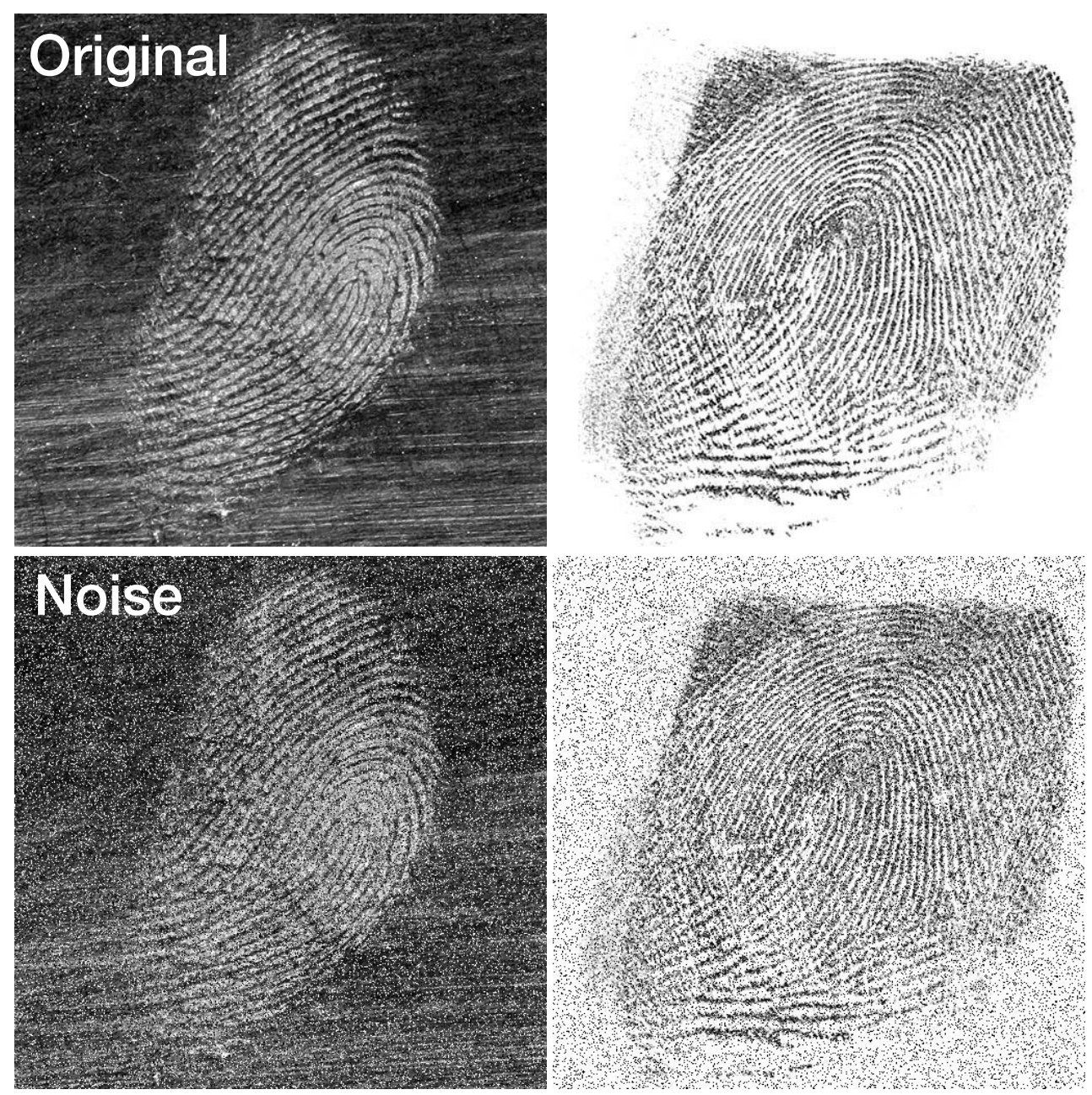

Figure 6. An example same-finger prints as original without any noise (top), and with $20 \%$ noise added to the images (bottom).

\section{Procedure}


The pre-experiment procedure was the same as in Experiment 1 with participants watching an instructional video describing the task and walking through an example same-finger and different-finger trial. We then presented participants with the 192 fingerprint pairs, one at a time, split into four blocks: Noisy print pairs with speed prompts, noisy print pairs with accuracy prompts, no noise print pairs with speed prompts, and no noise print pairs with accuracy prompts. We presented these blocks in a randomized order for each participant. For the two blocks emphasizing speed, we presented participants with "Speed Up" in black text during the inter-trial interval when they responded after five seconds of exposure to the prints. Participants also viewed a short video before each of the speed blocks instructing them to respond as quickly as possible for the next series of cases. For the two blocks emphasizing accuracy, participants were instructed to respond as accurately as possible, and were presented with "Slow Down" in black text during the inter-trial interval each time they responded before five seconds had elapsed. As in Experiment 1, participants were instructed to judge whether the two prints belong to the same finger or two different fingers using " $\mathrm{Z}$ " and "/" as response keys. The procedure for advancing through the trials was also the same as Experiment 1, with a 10 second response window and a 1 second interval between trials.

\section{Results}

We removed one participant with a non-response rate of $17.28 \%$. The non-response rates for the remaining 69 participants ranged between $0.00 \%$ and $8.85 \%$. Consistent with modeling of simple-choice tasks, we censored RTs less than $250 \mathrm{~ms}(0.75 \%$ of the data); more rapid RTs would be insufficient to make a valid decision.

\section{Discrimination Performance}

We used the same statistical procedures as outlined in Experiment 1 to analyze discrimination performance (correct and incorrect RTs, and accuracy). Predictors included stimulus type, noise (no noise vs. noise) and emphasis type (speed vs. accuracy). Statistical results are shown 
in Table 2. Correct and incorrect RTs quantiles $(0.1,0.5$, and 0.9$)$, and mean accuracy are shown in Figure 7.

As expected, there was a credible main effect of emphasis type on correct RTs and incorrect RTs, such that responses were considerably faster under speed emphasis compared to accuracy emphasis (correct: $2.10 \mathrm{~s}$ vs 5.31 ; incorrect: $2.20 \mathrm{~s}$ vs. $5.60 \mathrm{~s}$ ). While there was a credible two-way interaction between noise and emphasis type on correct RTs, the effect was not substantial; the difference in correct RTs between no-noise and noisy prints under speed emphasis was $0.14 \mathrm{~s}$, whereas the difference under accuracy emphasis was $0.08 \mathrm{~s}$. Incorrect RTs were credibly faster for different-finger prints compared to same-finger prints $(3.77 \mathrm{~s}$ vs. 3.90s). There was a credible interaction between stimulus type and noise on accuracy; for same-finger prints, accuracy declined for prints with noise compared to print without noise, whereas noise did not affect accuracy for different-finger prints. Finally, there was a credible, but weak interaction between stimulus type and emphasis-type on accuracy; whereas accuracy did not differ between emphasis types for same-finger prints, accuracy slightly improved for different-finger prints under accuracy emphasis compared to speed emphasis.
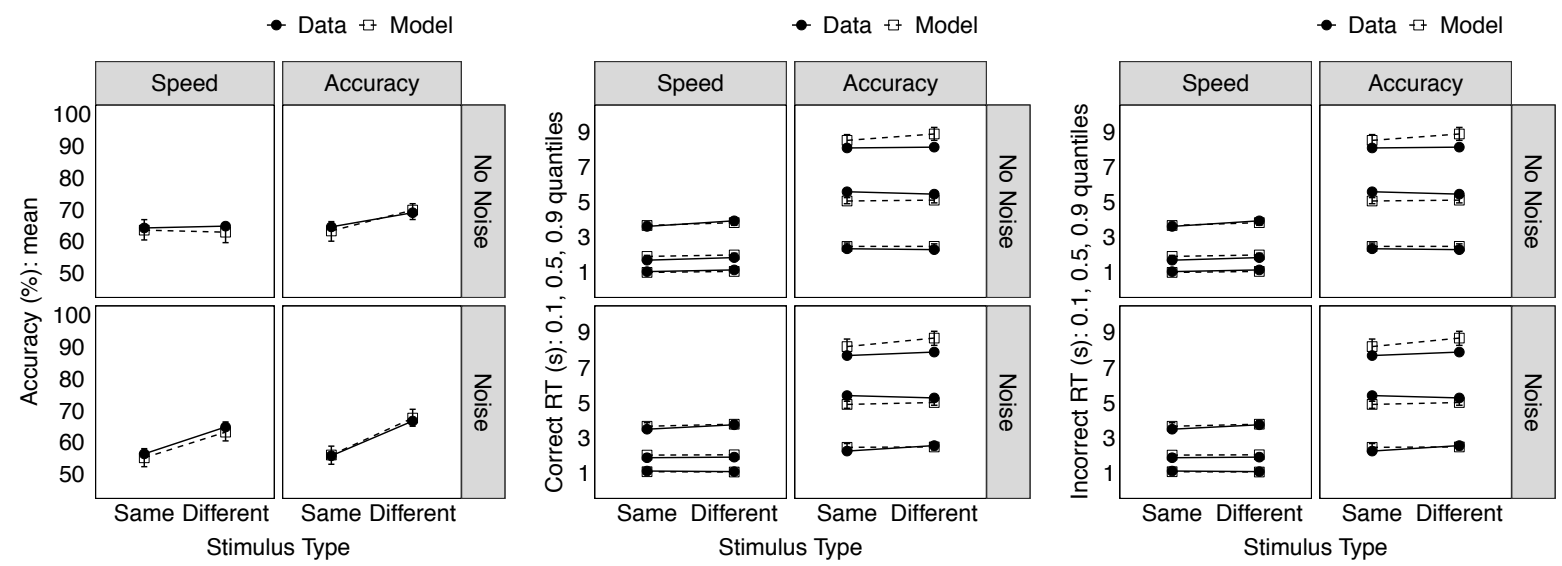

Figure 7. Experiment 2: Fits of the LBA to mean accuracy (left graph), and quantiles of correct (middle graph) and incorrect response times (right graph) of the fingerprint discrimination task. The bars show the 95\% quantiles of the posterior predictives.

\section{LBA Modeling and Analysis}


Selective influence - the idea that the effect of an experimental manipulation is captured by a single model parameter - has been important for the benchmarking of evidence accumulation models. A fundamental assumption of selective influence is that the effects of speed-accuracy emphasis is captured by the changes in the response caution (i.e., threshold). However, this assumption has recently been challenged. Using basic laboratory tasks, for example, Rae et al. (2014) found that as expected, individuals reduced their response threshold under a speed emphasis. They also found an increase in mean rate under accuracy emphasis and concluded that prioritizing accuracy produces an increase in the quality of information accumulated from the stimulus. Palada et al. (2018), however, have found some evidence for selective influence in complex tasks. They show that distinct determinants of time pressure had selective influence on cognitive processes; for example, in Study 2 the number of stimuli influenced threshold, whereas the time available influenced the rate at which information was accumulated.

In Experiment 2 we test whether the parameters respond to the experimental manipulations in a theoretically coherent way. We allow emphasis type to influence both threshold and mean rate. Initially, we also sought to test whether noise would affect threshold and mean rate. However, this model was unstable, so we had to simplify the model by constraining threshold across levels of the noise manipulation. We believe this assumption is reasonable because previous research has found that noise does not influence threshold. For example, Palada et al. (2016) used a similar noise manipulation and found that it affected inputs to the decision process (i.e., mean rate), but not the response threshold. The fit of the model after making this simplifying assumption was still very good. The model parameterization was otherwise consistent with Experiment 1, such that rate variability was influenced by the match factor and stimulus type, where non-decision time and start point variability were estimated across experimental conditions (see Table 1). 
We used the same modeling approach as Experiment 1. First, the model provided a good fit to mean accuracy and the distribution of correct and incorrect RTs (see Figure 7). Second, we examine the posterior estimates of model parameters to understand how the model explains the effects of noise and emphasis type manipulations on discrimination performance. The parameter estimates are shown in Figure 8. Table 3 presents the effects of the experimental manipulations and the accumulator related factors on model parameters. 


\section{Table 2.}

Experiment 2: Bayesian generalized linear mixed-model coefficients for discrimination task performance measures.

\begin{tabular}{lccc}
\hline Effect & Correct RT & Error RT & Accuracy \\
\hline $\mathrm{S}$ & -0.01 & 0.02 & -0.13 \\
& {$[-0.02,0.00]$} & {$[0.01,0.03]$} & {$[-0.17,-0.10]$} \\
$\mathrm{N}$ & 0.01 & 0.00 & -0.10 \\
& {$[0.00,0.02]$} & {$[-0.01,0.01]$} & {$[-0.14,-0.07]$} \\
$\mathrm{SA}$ & -0.48 & -0.50 & -0.04 \\
& {$[-0.49,-0.47]$} & {$[-0.51,-0.49]$} & {$[-0.07,0.00]$} \\
S.N & 0.01 & -0.01 & -0.08 \\
& {$[0.00,0.02]$} & {$[-0.02,0.01]$} & {$[-0.11,-0.04]$} \\
S.SA & -0.01 & 0.00 & 0.04 \\
& {$[-0.02,0.00]$} & {$[-0.01,0.01]$} & {$[0.00,0.07]$} \\
N.SA & 0.02 & 0.01 & 0.02 \\
& {$[0.01,0.02]$} & {$[0.00,0.03]$} & {$[-0.02,0.05]$} \\
S.N.SA & 0.01 & -0.01 & -0.01 \\
& {$[0.00,0.02]$} & {$[-0.02,0.01]$} & {$[-0.05,0.03]$} \\
\hline
\end{tabular}

Note. Experimental effects are stimulus type (S), noise (N), and emphasis type (SA); 95\% CIs are in square brackets.

Emphasis type had a credible effect on threshold, such that participants had a considerably higher threshold under accuracy emphasis compared to speed emphasis. There was a credible three-way interaction between stimulus type, noise and emphasis type on the differences between mean rate for the matching and mismatching accumulators. Under accuracy emphasis, the difference in mean rates was greater for different-finger prints compared to same-finger prints, and this effect was stronger for prints without noise compared to prints with noise. The same effect was observed under speed emphasis for prints with noise. For prints without noise, there was no difference in mean rates between samefinger prints and different-finger print. Finally, there was a credible two-way interaction between stimulus type and the match factor on the variability in the rate of evidence accumulation. Rate variability for the matching (i.e., correct) accumulator was greater than 
the rate variability for the mismatching (i.e., incorrect) accumulator for same-finger prints, whereas opposite effect occurred for different-finger prints.

The distribution of rates of evidence accumulation is shown in Figure 9. The graph shows how sensitivity did not appear to vary across emphasis and noise conditions for different-finger pairs. Unexpectedly, for same-finger prints presented without noise, sensitivity was greater for prints in the speed condition compared to the accuracy condition; this was driven by the difference in mean rates between the two accumulators. From the graphs it is clear that the decrease in sensitivity for same-finger pairs presented with noise compared to prints without noise was driven by the decrease in the differences between the two accumulators.

Overall, different-finger prints had greater accuracy compared to same-finger prints when prints were presented with noise, or when prints were presented without noise and under accuracy emphasis. The model captures this by the greater difference in mean rates between the two accumulators for different-finger prints compared to same fingerprints. However, the stimuli had comparable accuracy when presented without noise and under speed emphasis. The model also captures this by the comparable difference in mean rates between the two accumulators for both stimulus types. Emphasis type mainly affected response times and had a weak effect on accuracy. The considerably faster response times under speed emphasis compared to accuracy emphasis was captured by two mechanisms: The lower threshold under speed emphasis, as well as the greater overall rate of evidence accumulation. 

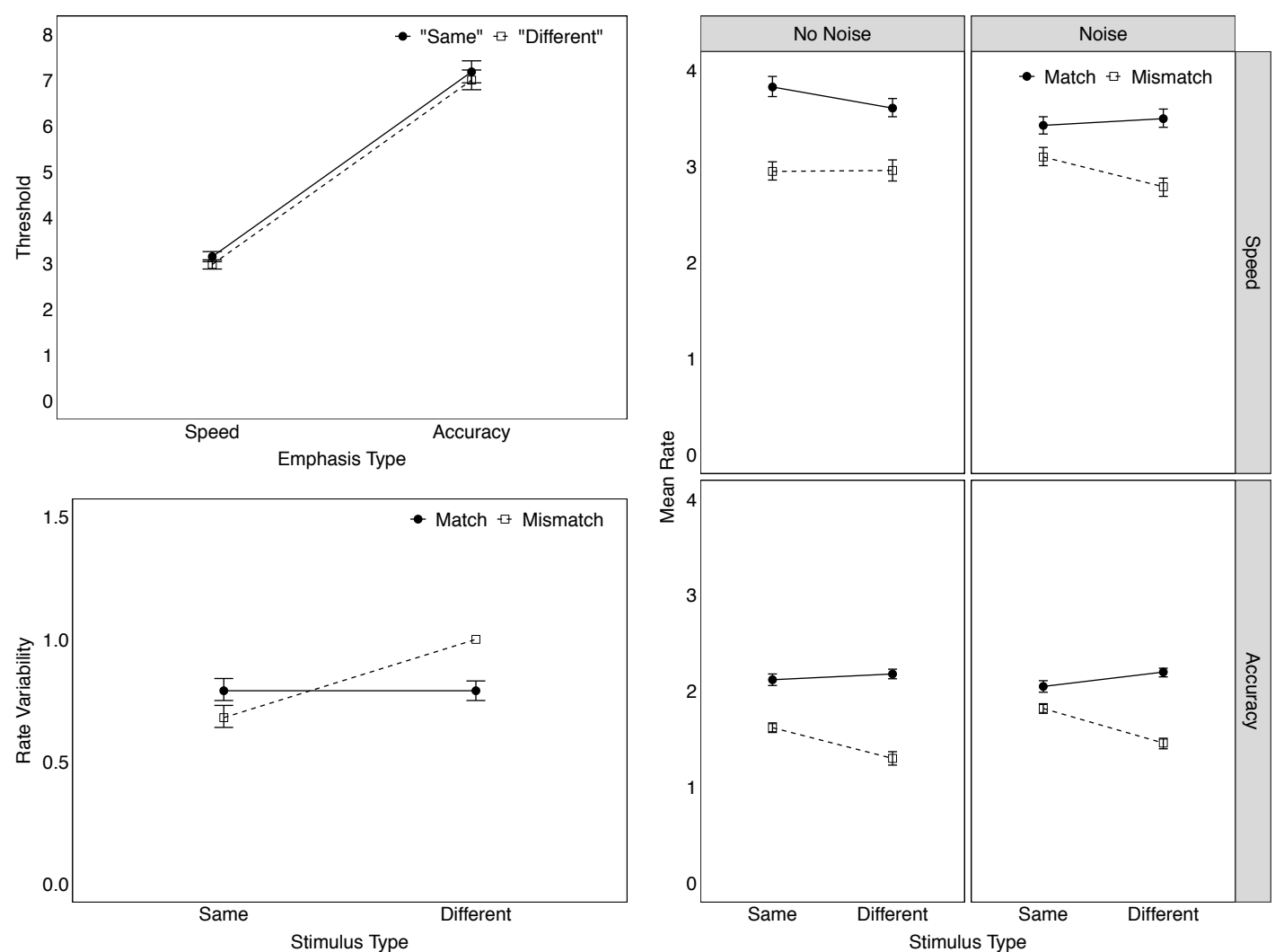

Figure 8. Experiment 2: Median parameters estimates for the LBA model, including response caution (top left panel), mean rate (right panel) and rate variability (bottom left panel). The bars show the $95 \%$ credible intervals. 
Speed Emphasis

Match (i.e., correct) $\square$ Mismatch (i.e., incorrect)

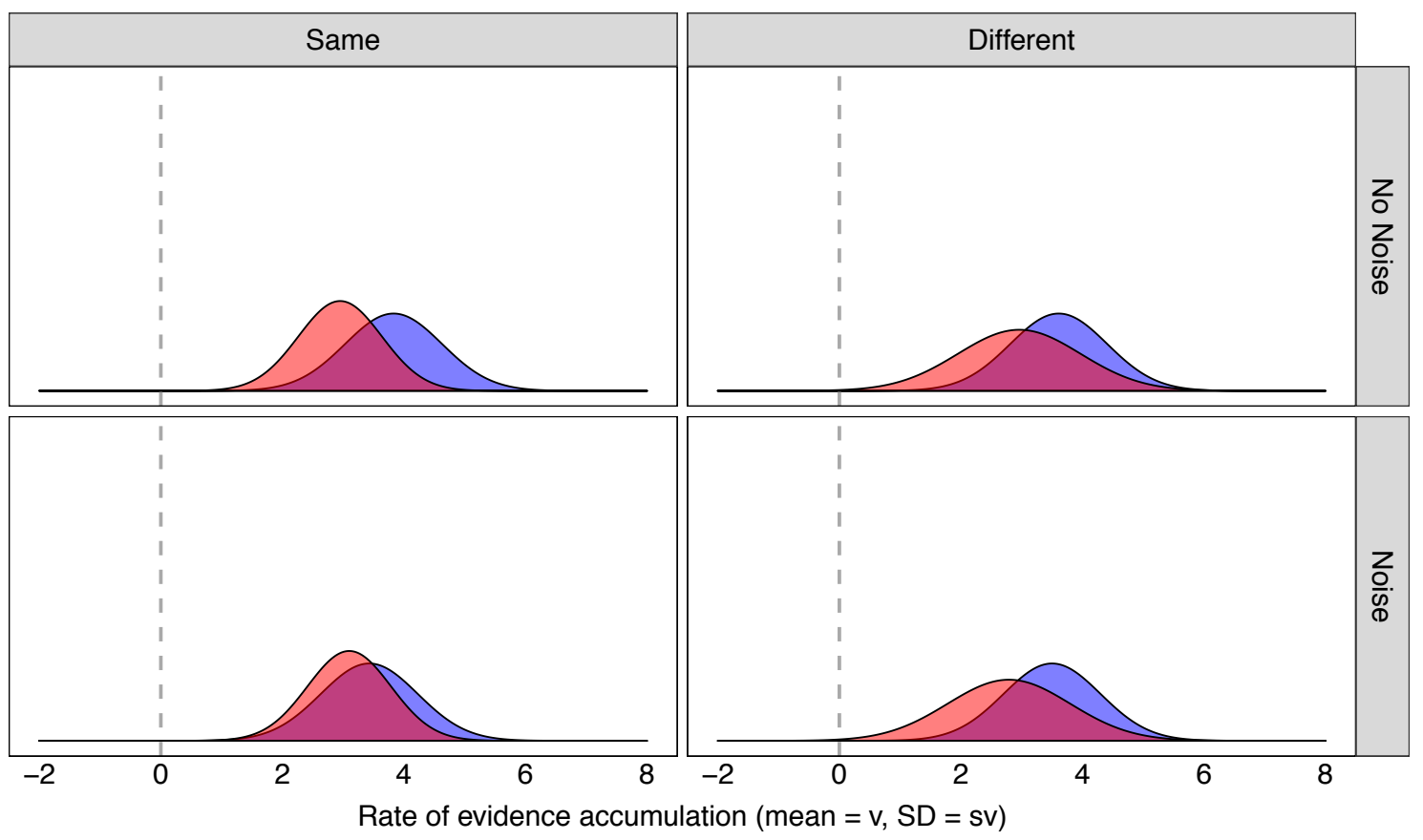

Accuracy Emphasis

Match (i.e., correct) $\square$ Mismatch (i.e., incorrect)
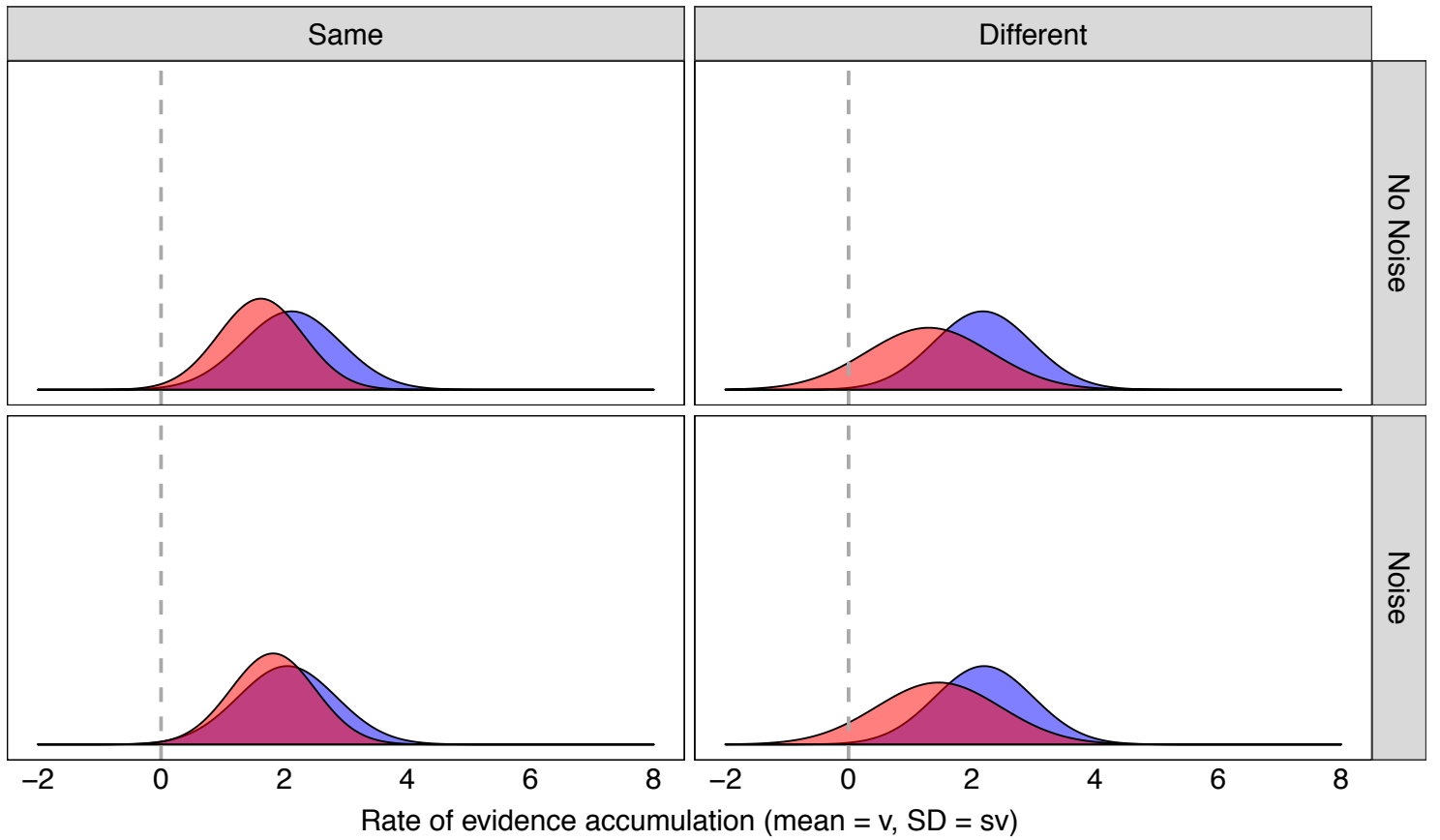

Figure 9. Experiment 2: Distribution plots of rates of evidence accumulation for matching and mismatching accumulators. The top four quadrants correspond to speed, while the bottom four quadrants correspond to accuracy emphasis. Rows alternate between prints without noise and with noise. Columns refer stimulus types. Distributions were generated using posterior medians. Vertical line reflects zero truncation of rate sampling. 
Table 3.

Experiment 2: Contrasts for experimental, response and match factor effects on LBA model parameters

\begin{tabular}{|c|c|c|c|}
\hline Effect & $\begin{array}{c}\text { Threshold } \\
(B)\end{array}$ & $\begin{array}{c}\text { Difference in Mean Rates } \\
\left(v_{\text {match }}-v_{\text {mismatch }}\right)\end{array}$ & $\begin{array}{l}\text { Rate Variability } \\
(s v)\end{array}$ \\
\hline S & - & $\begin{array}{c}-0.38 \\
{[-0.51,-0.25]}\end{array}$ & $\begin{array}{c}-0.16 \\
{[-0.19,-0.12]}\end{array}$ \\
\hline $\mathrm{N}$ & - & $\begin{array}{c}0.27 \\
{[0.17,0.37]}\end{array}$ & - \\
\hline SA & $\begin{array}{c}-4.02 \\
{[-4.20,-3.85]}\end{array}$ & $\begin{array}{c}-0.38 \\
{[-0.54,-0.23]}\end{array}$ & - \\
\hline $\mathrm{R}$ & $\begin{array}{c}-0.17 \\
{[-0.35,0.01]}\end{array}$ & - & - \\
\hline $\mathrm{M}$ & - & - & $\begin{array}{c}-0.05 \\
{[-0.09,-0.02]}\end{array}$ \\
\hline S.N & - & $\begin{array}{c}0.13 \\
{[-0.01,0.28]}\end{array}$ & - \\
\hline S.SA & - & $\begin{array}{c}-0.61 \\
{[-0.85,-0.36]}\end{array}$ & - \\
\hline S.M & - & - & $\begin{array}{c}0.32 \\
{[0.24,0.40]}\end{array}$ \\
\hline N.SA & - & $\begin{array}{c}-0.28 \\
{[-0.48,-0.20]}\end{array}$ & - \\
\hline R.SA & $\begin{array}{c}-0.01 \\
{[-0.33,0.31]}\end{array}$ & - & - \\
\hline S.N.SA & - & $\begin{array}{c}-0.48 \\
{[-0.77,-0.09]}\end{array}$ & - \\
\hline
\end{tabular}

Note. Factors are stimulus type (S), noise $(\mathrm{N})$, emphasis type (SA), response type $(\mathrm{R})$ and match type (M); 95\% CIs are in square brackets; $v_{\text {match }}$ and $v_{\text {mismatch }}$ refer to the values of the matching and mismatching accumulators, respectively. Dashes reflect cases where the model parameter was not allowed to vary by the effect (see Table 1 for model parameterisation)

\section{Discussion}

In this experiment, our aim was to determine whether the LBA could provide an accurate description of the effects of emphasis type (speed or accuracy) and visual noise (no noise vs. noise) on fingerprint discrimination decisions. We examined whether the relationships between model parameters and experimental manipulations would coincide with findings from simpler choice tasks. We hypothesised that emphasis type would influence parameters 
concerned with the termination of the decision process itself (i.e., threshold), whereas visual noise would influence the inputs to the decision process (i.e., the rate of evidence accumulation). Our hypothesis regarding noise was supported. Our hypothesis regarding emphasis type was partially supported - in line with recent evidence (e.g., Rae et al., 2014), we found that emphasis type affected mean rate in addition to affecting threshold.

We found that emphasis type affected response caution, or the quantity of evidence required to reach a decision. Participants reduced their response caution under speed emphasis compared to accuracy emphasis in order to accelerate the decision process, as the accumulator has a shorter distance to travel and reaches the threshold sooner. Contrary to Experiment 1, we did not find evidence of a bias to respond "different". We suspect that participants were not willing to adopt a "different" response bias under the experimental conditions as the bias would exacerbate the relatively poor performance for same-finger prints in this experiment, which was worse than in Experiment 1.

In line with recent studies (Rae et al., 2014), emphasis type also influenced mean rate. The overall mean rate of evidence accumulation, as indexed by the mean of the matching and mismatching accumulators was considerably higher under speed emphasis compared to accuracy emphasis. This suggests that participants processed evidence faster under the speed emphasis. This finding is consistent with Palada et al. (2018) who found that individuals increased their rate of evidence accumulation under tighter deadlines. Contrary to Rae et al. (2014), we did not find evidence that the quality of evidence, as indexed by the difference between the two accumulators, increased under accuracy emphasis compared to speed emphasis. However, we provide evidence for Rae et al.'s conclusion that whether emphasis type primarily affects the speed or quality of evidence accumulation depends on whether the manipulation largely affects responses times or accuracy, respectively.

As expected, we found that noise negatively influenced discriminability, though this only occurred for same-finger prints. The decrease in discriminability was driven by the 
difference in mean rates between the two accumulators and captured the decline in accuracy for same-finger prints presented with noise compared to prints presented without noise. In contrast, noise did not affect the discriminability of different-finger pairs, which explains why no change in accuracy was observed between noise conditions. In line with our interpretation of the results from Experiment 1, we suspect that the between-finger differences inherent in the materials used in this experiment explain why we did not observe an effect of noise on different-finger discriminability. Specifically, the asymmetrical effects of noise on discriminability may have resulted from the noise manipulation being sufficiently strong to obscure similarities among same-finger prints (thereby decreasing discriminability for these pairs), but not sufficiently strong to obscure differences between different-finger prints (thereby failing to reduce discriminability for these pairs).

Overall, Experiment 2 suggests that the LBA model parameters can be interpreted meaningfully in the context of fingerprint discrimination. Consistent with studies using basic tasks (e.g., Rae et al. 2014), emphasis type influenced both response caution and rate of evidence accumulation (albeit in the opposite direction to what some previous research has found), whereas noise selectively influenced discriminability.

\section{Experiment 3}

In Experiment 1 and 2, we showed that the LBA can accurately capture fingerprint discrimination performance, and that model parameters can be mapped meaningfully to the underlying cognitive processes they are thought to reflect. In particular, speed-accuracy emphasis influenced response caution in a manner consistent with theory and previous research. Noise also reduced discriminability, but only for same-finger pairs. In Experiment 3, we examine how novices' decision-making processes evolve over time by using the LBA to quantify any change in decision processes after a feedback training intervention. There are a number of ways that training could affect decision processes. First, feedback may alter response biases as participants learn to adjust their prior expectations of stimuli. Second, 
feedback may improve accuracy by leading to increased response caution. Alternatively, feedback may improve accuracy by enhancing the quality of evidence that is accumulated as the participants learn to attend to more diagnostic print features - an effect that would be mediated by the rate parameters (i.e., the mean or variability in the rate of evidence accumulation). In this experiment, we test for these possible effects by allowing model parameters to vary with group type (training vs. no training) and block (pre- vs. post-training).

\section{Method}

\section{Participants \& Stimuli}

A third group of seventy psychology undergraduates (21 male and 48 female) with an average age of $20.13(\mathrm{SD}=3.88)$ from The University of Queensland participated in Experiment 3 in return for course credit. The image-set was identical to Experiment 1, and we generated 192 fingerprint pairs (96 same-finger pairs and 96 different-finger pairs) for each participant using the same method. There were 32 same-finger pairs and 32 different-finger pairs print pairs in each of the pre-test, training, and post-test conditions.

\section{Procedure}

Participants were randomly assigned to either the feedback or no feedback condition. The preexperiment procedure was the same as in Experiment 1 and Experiment 2; participants watched an instructional video, which consisted of the task description and an explanatory example of both same-finger and different-finger pairs. Participants were then presented with 192 pairs of fingerprints, one at a time, in three blocks of trials. In the first block, the pre-test phase of the experiment, 64 pairs of fingerprints were presented. In the second block, the training phase a new set of 64 fingerprints were presented; feedback participants were presented with "Correct" in green text, or "Incorrect" in red text, corresponding to whether they correctly classified the pair of fingerprints and no feedback participants viewed a blank screen between trials. In the third block, the post-test phase, a new set of 64 fingerprints were presented without feedback. As in Experiments 1 and 2, participants were instructed to 
classify the prints as originating from the same finger with the " $Z$ " key, or from different fingers using the "/" key.

\section{Results}

Non-response rates across participants ranged between $0.00 \%$ and $5.21 \%$. No participants were removed from analyses because none had a non-response rate greater than $10 \%$.

However, five responses less than $250 \mathrm{~ms}$ were censored.

\section{Discrimination Performance}

We used the same procedure as Experiment 1 to analyze Experiment 3 performance. The experimental factors included stimulus type, block (pre-training vs. post-training) and feedback group (feedback vs. no feedback). Results are shown in Table 4 and Figure 10. There was a credible three-way interaction on accuracy. For those in the no-feedback group, accuracy was stable across blocks for both stimuli. In contrast, for those in the feedback group, accuracy improved for same-finger prints after receiving training, whereas accuracy slightly decreased for different-finger prints. There was a credible effect of block on correct and error RTs, such that RTs were faster at post-test compared to pre-test (correct RTs: $2.74 \mathrm{~s}$ vs $3.57 \mathrm{~s}$; error RTs: $3.30 \mathrm{~s}$ vs $4.10 \mathrm{~s}$ ). Error RTs were also faster for different-finger prints $(3.59 \mathrm{~s})$ than same-finger prints $(3.80 \mathrm{~s})$. 
Table 4.

Experiment 3: Bayesian generalized linear mixed-model coefficients for task performance

\begin{tabular}{lccc}
\hline Effect & Correct RT & Error RT & Accuracy \\
\hline S & 0.01 & 0.04 & -0.14 \\
& {$[0.00,0.03]$} & {$[0.02,0.06]$} & {$[-0.19,-0.09]$} \\
B & -0.15 & -0.13 & 0.03 \\
& {$[-0.16,-0.13]$} & {$[-0.15,-0.11]$} & {$[-0.01,0.08]$} \\
G & -0.06 & -0.05 & 0.01 \\
& {$[-0.12,0.01]$} & {$[-0.13,0.03]$} & {$[-0.05,0.08]$} \\
S.B & 0.00 & 0.01 & 0.08 \\
& {$[-0.02,0.01]$} & {$[-0.01,0.03]$} & {$[0.03,0.12]$} \\
S.G & 0.00 & 0.00 & 0.04 \\
& {$[-0.01,0.02]$} & {$[-0.02,0.02]$} & {$[-0.01,0.08]$} \\
B.G & -0.01 & 0.01 & -0.01 \\
& {$[-0.03,0.00]$} & {$[-0.01,0.03]$} & {$[-0.06,0.03]$} \\
S.B.G & -0.01 & 0.00 & 0.10 \\
& {$[-0.02,0.00]$} & {$[-0.01,0.02]$} & {$[0.05,0.14]$} \\
\hline
\end{tabular}

Note. Experimental effects are stimulus type (S), block (B) and group type (G); 95\% CIs are presented in square brackets.
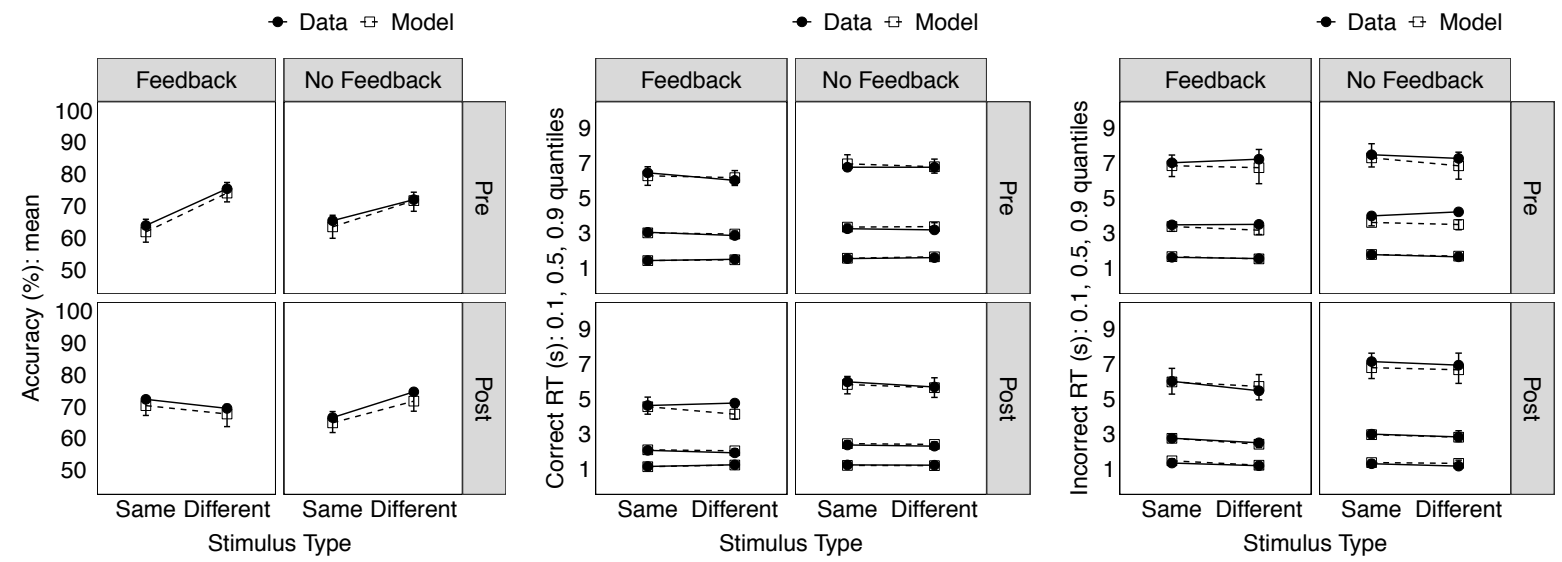

Figure 6. Experiment 3: Fits of the LBA to mean accuracy (left graph), and quantiles of correct (middle graph) and incorrect response times (right graph) of the fingerprint discrimination task. The bars show the 95\% credible intervals of the posterior predictives. 


\section{LBA Modeling and Analysis}

We used the LBA to examine how the processes underlying fingerprint discrimination change over time and in response to feedback. The model parameterisation is shown in Table 1 . We allow threshold and mean rate to vary with block and training, and also allow mean rate to vary between stimuli. As we are interested in the general effects of training on the inputs to the decision processes (i.e., rate parameters), we also allow rate variability to vary with the experimental factors. The estimates of non-decision time and start point variability were constrained across experimental conditions, such that only a single value was estimated for each parameter (see Table 1). We used the same modeling approach as in Experiments 1 and 2. However, since we fit models separately for each feedback group (as feedback group was manipulated between participants), non-decision time $(t 0)$, start point variability $(A)$, and rate variability ( $s v)$ also varied across feedback conditions. As can be seen in Figure 10, the model provides an excellent fit to the data for both feedback groups.

Figure 11 displays the estimates of response caution, mean rates, and variability in the rate of evidence accumulation. We used the same procedure as Experiments 1 and 2 to analyze the results, with experimental and accumulator related effects shown in Table 5 . For response caution, all two-way interactions were credible, though there was an interesting trend when considering all three factors (group, block, and response type). At pre-test, both groups set a lower threshold to respond "different" compared to the threshold to respond "same". For the no-feedback group, the strength of this effect weakened at post-test, such that the threshold became more similar between the response options. For the feedback group, the effect reversed, such that participants set a higher threshold to respond "same" compared to the threshold to respond "different". At post-test, the feedback group was also generally more cautious than the no-feedback group.

There was a credible three-way interaction between block, group, and stimulus type on the difference in mean rates. At pre-test, both groups had a difference in mean rates that was 
higher for different-finger prints compared to same-finger prints. At post-test, after having received feedback, the difference in mean rates became more similar between the two types of stimuli, as the difference between mean rates improved for same-finger prints. For the nofeedback group, the difference in mean rates for same-finger prints did not change from pretest to post-test, whereas the difference for different-finger prints increased.

There were credible main effects of block, stimulus, and match factor on the variability in rate of evidence accumulation. Rate variability was higher at post-test compared to pre-test, for different-finger prints compared to same-finger prints, and for the matching (i.e., correct) accumulator than for the mismatching (i.e., incorrect accumulator). The four-way interaction was not credible. However, Figure 11 shows that the patterns in rate variability were similar between groups at pre-test, whereas there was some evidence of group differences at post-test. We tested for this by comparing post-test rate variability between accumulators for each stimulus type. For same-finger pairs, the difference in rate variability between accumulators did not differ between groups $(-0.05[-0.71,0.58])$. For different-finger pairs, the difference in rate variability between accumulators was greater for the feedback group compared to the non-feedback group due to the greater match variability for the feedback group $(-1.00[-1.67,-$ $0.34])$.

The distribution of rates of evidence accumulation is shown in Figure 12. The graph shows how sensitivity was similar across groups at pre-test. At post-test, any improvement in sensitivity afforded by the greater difference in means between the distributions was offset by the increase in variance. As a result, training did not appear to influence overall sensitivity. Therefore, the improvement in accuracy for same-finger prints and drop in accuracy for different-finger prints for the feedback group resulted from the reversal in response bias from pre-test to post-test. The generally faster response times from pre-test to post-test was captured by the increase in mean rates, as the accumulators travel fasters towards the threshold. 
Table 5.

Experiment 3: Contrasts for experimental, response and match factor effects on LBA model parameters

\begin{tabular}{|c|c|c|c|}
\hline Effect & $\begin{array}{l}\text { Threshold } \\
\qquad(B)\end{array}$ & $\begin{array}{c}\text { Difference in Mean Rates } \\
\left(v_{\text {match }}-v_{\text {mismatch }}\right)\end{array}$ & $\begin{array}{l}\text { Rate Variability } \\
\qquad(s v)\end{array}$ \\
\hline $\mathrm{S}$ & 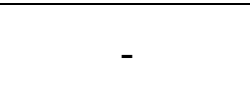 & $\begin{array}{c}-0.63 \\
{[-0.91,-0.36]}\end{array}$ & $\begin{array}{c}-0.13 \\
{[-0.25,-0.02]}\end{array}$ \\
\hline B & $\begin{array}{c}-0.62 \\
{[-0.79,-0.45]}\end{array}$ & $\begin{array}{c}0.29 \\
{[0.09,0.49]}\end{array}$ & $\begin{array}{c}-1.12 \\
{[-1.26,-0.98]}\end{array}$ \\
\hline G & $\begin{array}{c}0.02 \\
{[-0.18,0.13]}\end{array}$ & $\begin{array}{c}-0.52 \\
{[-0.88,-0.15]}\end{array}$ & - \\
\hline $\mathrm{R}$ & $\begin{array}{c}0.26 \\
{[0.13,0.38]}\end{array}$ & - & - \\
\hline M & - & - & $\begin{array}{c}1.04 \\
{[0.92,1.17]}\end{array}$ \\
\hline S.B & - & $\begin{array}{c}-0.08 \\
{[-0.39,0.23]}\end{array}$ & -5 \\
\hline S.G & - & $\begin{array}{c}-0.45 \\
{[-1.00,0.10]}\end{array}$ & - \\
\hline B.G & $\begin{array}{c}-1.11 \\
{[-1.45,-0.77]}\end{array}$ & $\begin{array}{c}-0.76 \\
{[-1.16,-0.37]}\end{array}$ & - \\
\hline B.R & $\begin{array}{c}0.62 \\
{[0.37,0.88]}\end{array}$ & - & - \\
\hline G.R & $\begin{array}{c}0.50 \\
{[0.25,0.75]}\end{array}$ & - & - \\
\hline S.B.G & - & $\begin{array}{c}-1.06 \\
{[-1.68,-0.44]}\end{array}$ & - \\
\hline R.B.G & $\begin{array}{c}-0.24 \\
{[-0.75,0.25]}\end{array}$ & - & - \\
\hline S.B.G.M & 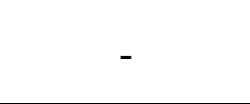 & - & $\begin{array}{c}0.69 \\
{[-0.36,1.75]}\end{array}$ \\
\hline
\end{tabular}

Note. Factors are stimulus type (S), block (B), group type (G), response type (R) and match type (M); 95\% CIs are in square brackets; $v_{\text {match }}$ and $v_{\text {mismatch }}$ refer to the values of the matching and mismatching accumulators, respectively. Dashes reflect cases where the model parameter was not allowed to vary by the effect (see Table 1 for model parameterisation) 

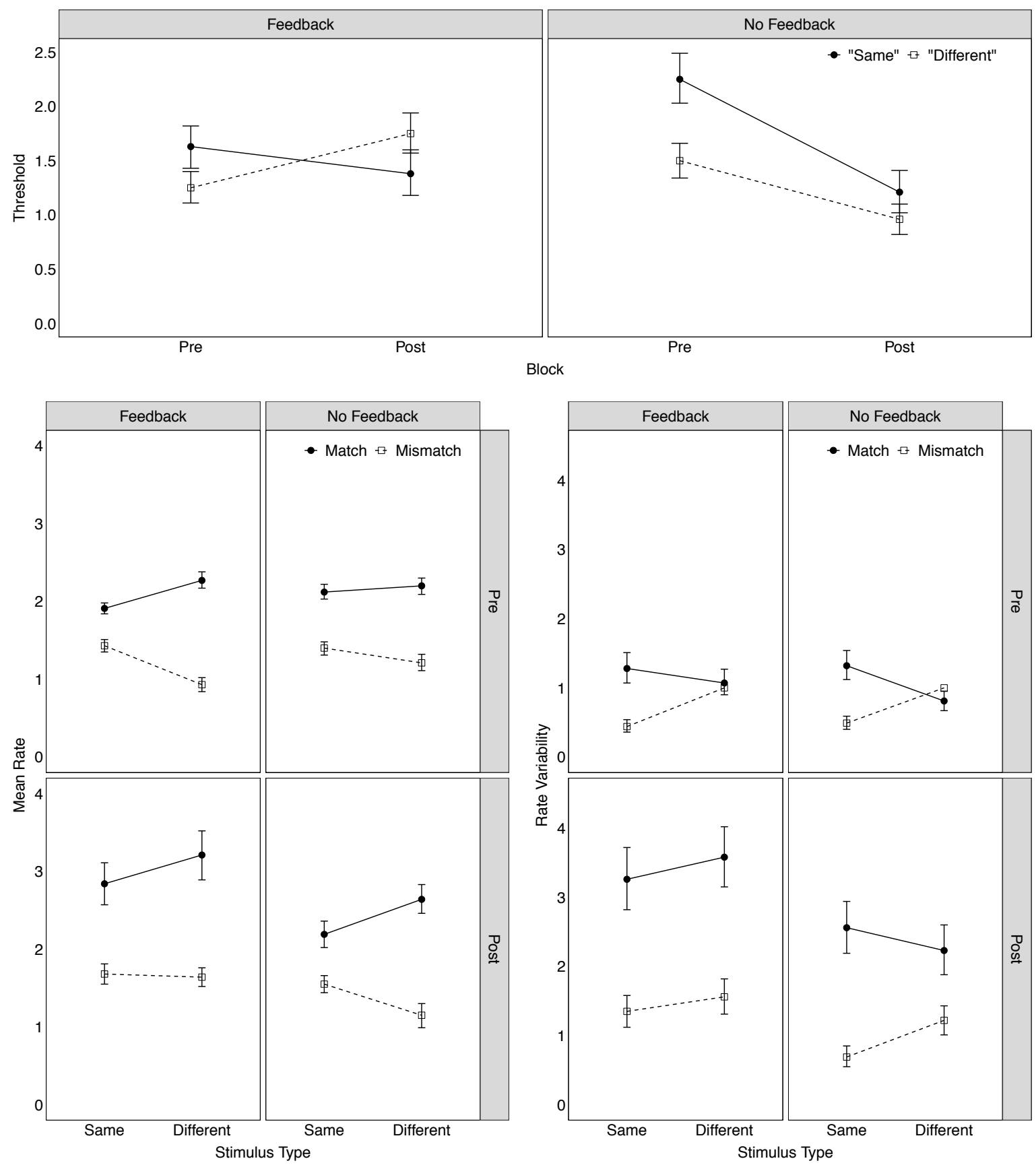

Figure 7. Experiment 3: Median parameters estimates for the LBA model, including response caution (upper-left panel), mean rate (lower-left panel) and rate variability (right panel). The bars show the $95 \%$ credible intervals. 


\section{No Feedback}

Match (i.e., correct) $\square$ Mismatch (i.e., incorrect)

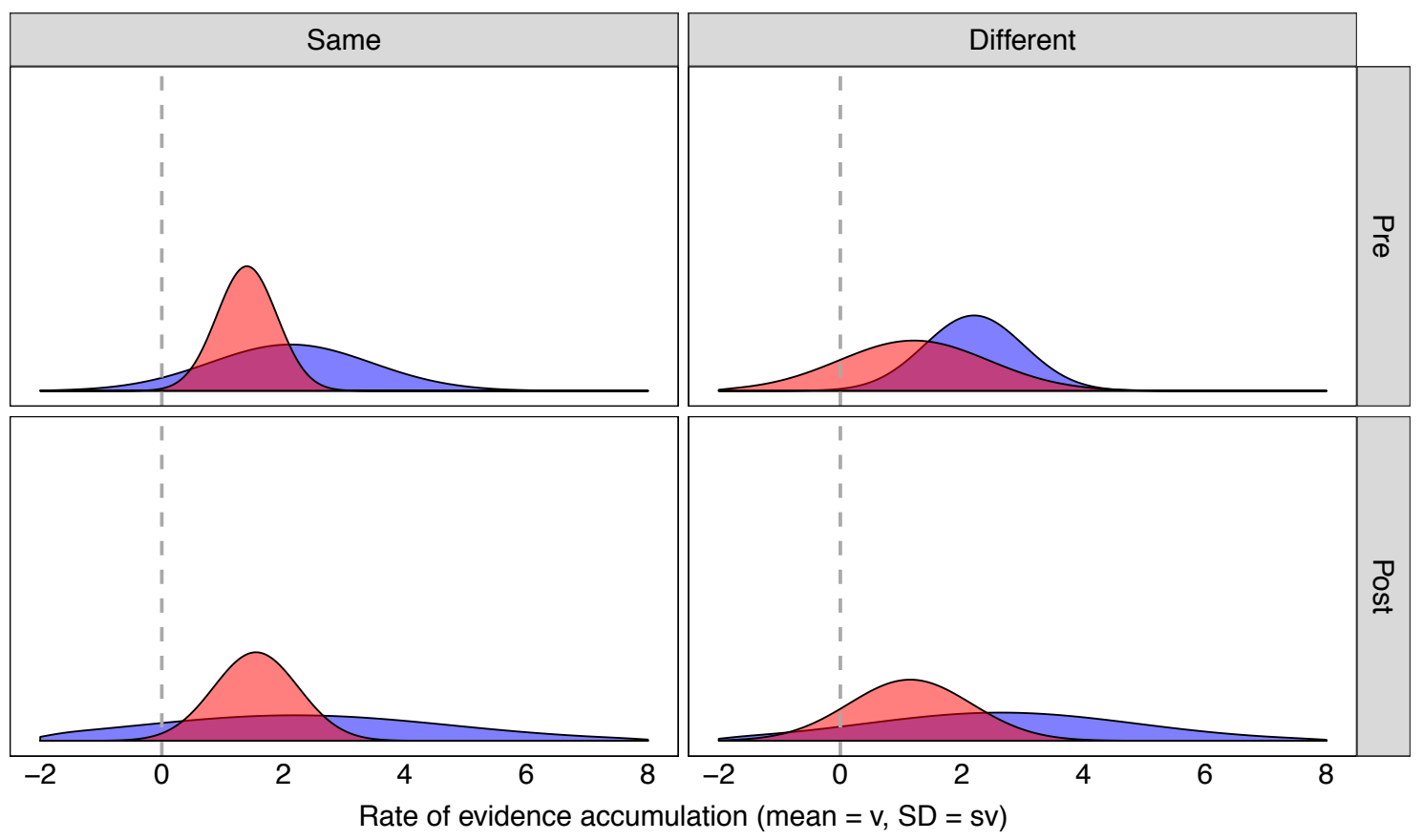

Feedback

Match (i.e., correct) $\square$ Mismatch (i.e., incorrect)
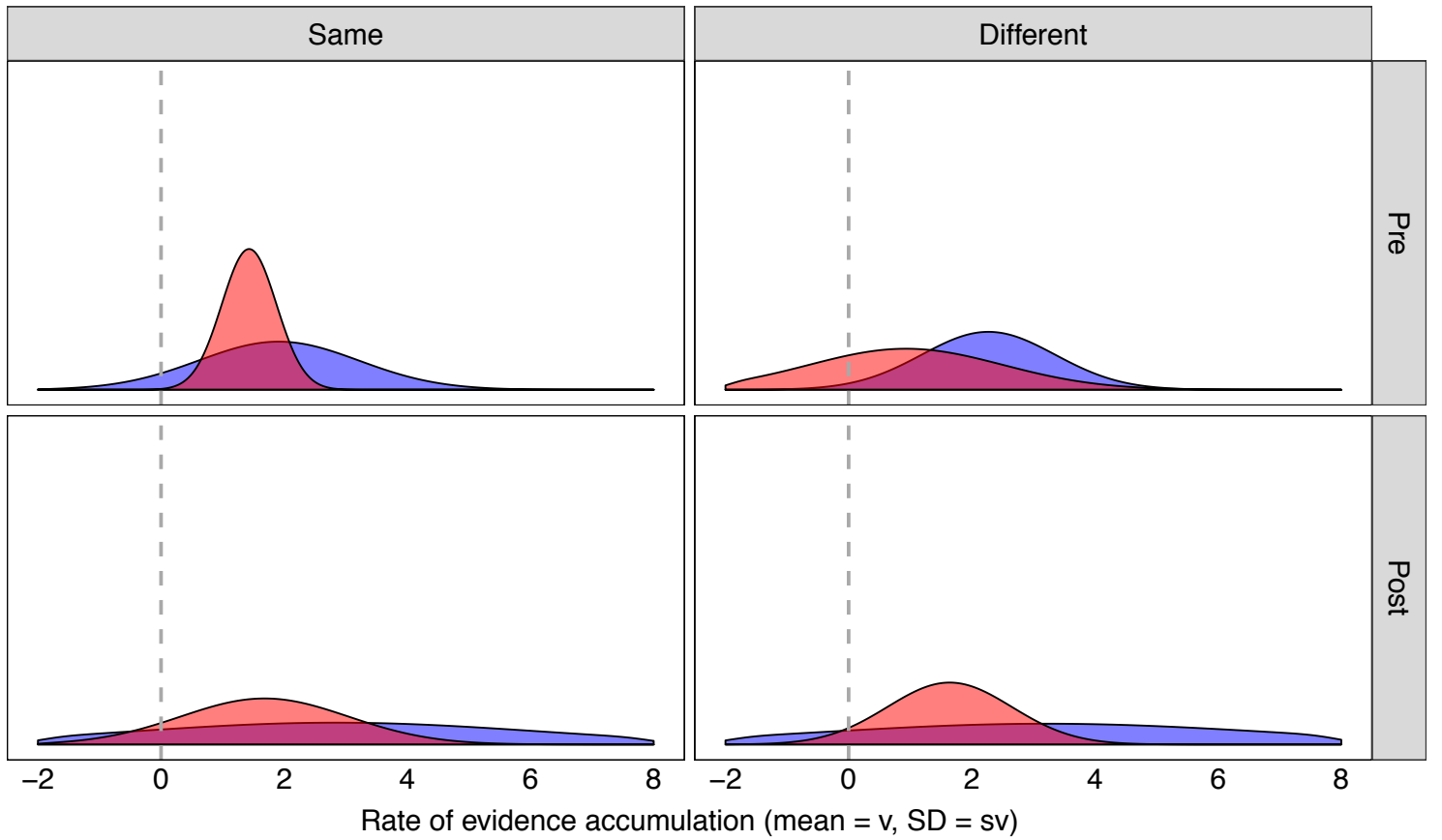

Figure 8. Experiment 3: Distribution plots of rates of evidence accumulation for matching and mismatching accumulators. The top four quadrants show the no feedback group, while the bottom four quadrants show the feedback group. Rows alternate between pre and post training trials. Columns refer stimulus types. Distributions were generated using posterior medians. Vertical line reflects zero truncation of rate sampling. 


\section{Discussion}

In this experiment, we used the LBA to understand the cognitive processes underlying the effects of a training intervention on fingerprint discrimination. The behavioral data revealed that training improved same-finger pair accuracy compared to baseline irrespective of the considerable decrease in response times, whereas different-finger pair accuracy slightly decreased after receiving training. As expected, accuracy did not improve over trial phases for the no-feedback group, though response times decreased. Our modeling suggests that the effects of feedback on performance is mediated by a number of cognitive processes, including response caution and rate variability.

In line with Experiment 1, at pre-test, both groups had a bias to respond "different" by setting a lower response threshold than the "same" response threshold. The no-feedback group maintained this response bias at the post-test phase, though to a much lesser extent. We suspect that this decline in response bias for the no-feedback group may have occurred as, over the course of the experiments, participants may have become aware of the relative frequency with which they responded "different" compared to "same" and sought to balance their response frequency between the response alternatives. The feedback group, by contrast, reversed their response bias after having received training. In this case, the feedback provided in the training phase may have made participants aware of their lower accuracy for samefinger pairs compared to different-finger pairs. The reversal of the response bias may reflect an attempt to compensate for this discrepancy in accuracy. This observation is consistent with Palada et al. (2018), who found that individuals adopted a response bias corresponding to the choice with poorer accuracy. This observation is also consistent with prior signal detection modelling of novices' fingerprint discrimination decisions (Searston, Tangen \& Eva, 2015), showing opposite patterns of response bias across experiments with and without feedback.

There was some evidence that training had an effect on the inputs to the evidence accumulation process, more specifically on the variability of evidence accumulation. Rate 
variability generally increased from pre-test to post-test. There was a trend for this effect being stronger for the feedback group. Specifically, the rate variability for the match accumulator for different-finger prints increased after having received training, such that it became greater than the rate variability for the match accumulator of same-finger prints. It seems unlikely that this was an artifact of the stimuli, as heterogeneity was similar across pretest and post-test blocks. Rather, this pattern of results suggests that training may have led to a strategic change in the information that was attended to when discriminating prints. The change in processing appears to have negatively influenced the effectiveness in processing different-finger pairs, and this could also explain why participants reversed their response bias post-feedback from favoring a "same" finger response to a "different" finger response.

\section{General Discussion}

Our aim was to model the dynamics of decisions about whether pairs of fingerprints came from the same finger or different fingers. To better understand the underlying decision process, we modeled choices and response times on a fingerprint discrimination task using a standard evidence accumulation model—-linear ballistic accumulation (Brown \& Heathcote, 2008). Forensic decision-making about the source of a crime-scene trace, such as a fingerprint, relies on the perceptual sensitivity of human examiners to distinguish specimens that match from those that do not match. Signal detection models have helped to better quantify the conditions that affect forensic examiners' discriminability separately from their response bias (Thompson et al. 2013a; Searston \& Tangen, 2017a, 2017b). However, forensic science is fundamentally an evidence accumulation process, and forensic examiners report taking a great deal of care and time in making their decisions so as to avoid errors. Evidence accumulation models offer an opportunity to gain new insights about the underlying cognitive processes of experts by accounting for the time course of their decisions. As a first step, we sort to test the validity of the LBA as a model of fingerprint discrimination in three experiments with novices. 
The aim of Experiment 1 was to conduct an initial evaluation of the whether the LBA could account for the patterns of choice and response times on a fingerprint discrimination task. Overall, the model provided a very good account of the data, and yielded insights about the underlying decision process that would not have been clear from an inspection of the accuracy and response time data alone. Our analysis revealed, for instance, that the observed difference in accuracy between our same-finger and different-finger prints is likely due to the combination of two processes. First, people were biased toward classifying pairs as having originated from different fingers (i.e., they had a lower threshold for the "different finger" response). Second, people processed information more effectively when examining prints that had originated from different fingers compared to prints from the same finger. This finding contrasts with previous studies showing a liberal response bias toward "same" decisions among novices (e.g., Tangen, Thompson \& McCarthy, 2011; Thompson et al. 2013b; Searston, Tangen \& Eva, 2016). Since these previous studies used highly similar distractor prints resembling those often encountered in operational contexts, this discrepancy in novices' response biases across studies may be explained by our random pairing of different-finger pairs. That is, we used dissimilar different-finger pairs as opposed to the similar differentfinger pairs used in previous research, such that our set were more distinctively different.

The aim of Experiment 2 was to examine how components of the decision process change when speed or accuracy are emphasized and when perceptual noise is added to the stimulus. As expected, people were more cautious in their responding (i.e., set higher response thresholds) when accuracy was emphasized compared to when speed was emphasized. We did not observe a "different" response bias as found in Experiment 1 and in some conditions in Experiment 3. We took this lack of difference as evidence to suggest that the bias may not be robust under more difficult or time pressured contexts, as adopting a "different" bias would have decreased accuracy with the same-finger stimuli. Indeed, this explanation is consistent with previous findings demonstrating that novice and expert 
examiners display a neutral response bias when discriminating fingerprints presented for just 400 milliseconds (Searston \& Tangen, 2017b).

We also replicated the finding in Experiment 1 that discriminability is higher when viewing prints that originated from different fingers. This effect was robust to the presence of noise. In contrast, and as expected, discriminability for same-finger prints decreased when noise was introduced. In line with recent evidence (e.g., Rae et al., 2014), we found that emphasis type also influenced mean rate. Because our manipulation of emphasis type affected response times considerably more than it affected accuracy, the effects of emphasis type manifested through the overall rate at which evidence was accumulated, rather than the differences in rates of evidence accumulation between the matching and mismatching accumulators.

For prints that had originated from the same finger, discriminability decreased under accuracy emphasis compared to speed emphasis. This latter result was unexpected and may highlight a unique feature of crime-scene prints. The additional processing afforded under accuracy emphasis may negatively influence the discrimination of prints of the same finger because the individual further processes contextual information (e.g., distortion or pressure) and erroneously takes this as evidence for a different-finger pair. Under speed emphasis, the individual may only process information which is at least partially diagnostic of the samefinger pair, and so accuracy is less affected compared to when accuracy is emphasized. This conclusion is consistent with Rae et al. (2014), who suggest that emphasis type may influence the kind of information accumulated during the decision process. Speed emphasis, for instance, may focus attention on the more global or distributed characteristics of fingerprints (e.g., Searston \& Tangen, 2017a) that can be gleaned quickly at a glance or in noise (e.g., Thompson \& Tangen, 2014). Accuracy emphasis, on the other hand, may focus attention more on the finer features such as the 'minutiae' of a fingerprint. Directly examining the 
nature of the information that is extracted while processing fingerprints is a promising avenue for future research.

The aim of Experiment 3 was to examine how the components of the fingerprint discrimination process change in response to a training intervention where participants are given feedback regarding the correct response. The results for the pre-intervention block replicated Experiment 1, with people showing a bias toward discriminating pairs as originating from different fingers, and also processing information more efficiently when pairs came from different fingers. The training intervention had the effect of reversing this bias: Those who received the intervention showed a bias toward discriminating pairs as originating from the same finger, as opposed to originating from different fingers, during the post-intervention block. We suspect that the response bias reversed after training because participants attempted to compensate for their poorer accuracy in identifying pairs as originating from the same finger. This result is consistent with prior studies on novices' fingerprint discrimination decisions showing a liberal ("same") response bias in the absence of feedback, but a conservative (“different”) response bias in the presence of feedback (Searston, Tangen \& Eva, 2016).

Training did not improve overall discriminability. However, there was some evidence that training influenced the variability in the rate of evidence accumulation associated with making correct decisions, particularly for pairs that came from different fingers. We hypothesize that training may have led individuals to change how they processed pairs of prints. From training, individuals may have learned that differences in the images due to factors such as pressure, distortion, and completeness do not necessarily diagnose prints as originating from different sources. As a result, individuals may have attended to a greater range of information to inform their decisions, and this would have had a greater impact on the decision inputs to the pairs originating from different fingers. 
Across all three experiments, we observed an interesting difference in the variability of the rate of evidence accumulation between pairs originating from the same versus different fingers. In general, the variability in the rate of evidence accumulation for the matching accumulator was greater for pairs originating from the same finger than for pairs originating from different fingers. We argue that the difference in rate variability is due to inherent features of the stimuli. Different-finger pairs can often be identified fairly easily, by seeking out particular features that, if found, quickly diagnose the pair as originating from different fingers. This makes the rate of evidence accumulation fairly reliable, with less heterogeneity across different fingers resulting in lesser variability in the rate of evidence accumulation to make a correct decision. Indeed, such an interpretation is consistent with the original notion that rate variability captures heterogeneity in stimulus difficulty (Ratcliff, 1978), and recent evidence using heterogeneous multi-attribute stimuli (Palada et al., 2018). An experiment measuring rate variability across systematic changes in the similarity of same and different fingerprint pairs could be an interesting test of this idea in the future.

Overall, our findings suggest that the LBA can accurately reproduce the empirical patterns of choices and responses times observed across the three experiments in the fingerprint discrimination task. The effects of the manipulations on the model parameters, however, raise questions about the underlying decision process that warrant further research. The pattern of results suggests that novices may attend to extraneous information which is not diagnostic in deciding whether the prints originated from the same or different fingers. Our initial evidence from Experiment 3 suggests that perhaps novices can shift this strategy, though future research should attempt to further understand what information is processed in fingerprint discrimination. In line with this possibility, our modeling suggests that the LBA can be used to describe the effects of training on fingerprint discrimination, which could be used to inform training interventions. Our results also point to the idea that the contextual 
information presented in crime-scene prints can induce biases, which has implications for fingerprint discrimination in the field (Dror, 2018).

We believe the LBA is a fruitful avenue for understanding decisions involving naturalistic stimuli because it can model the dynamics of decision making. We have tested the model in a decision-making task that bears strong resemblance to the task in the wild, and to the materials that fingerprint examiners encounter in practice. The idiosyncratic contextual factors of the task and the stimuli did not pose a major issue for the evidence accumulation model, in that it was able to provide an accurate description of performance. Overall, our experiments continue to extend evidence supporting the wider application of evidence accumulation to applied tasks which have a close resemblance to their naturalistic inspiration (e.g., Palada et al., 2018). Further application of evidence accumulation models to fingerprint discrimination could help us better understand sources of bias in people's decision making, whether they pose a concern for discriminability, and how to calibrate them. Moreover, the evidence accumulation framework can help to understand how the decision-making process changes as people transition from novice to expert. The application of evidence accumulation models to other domains of perceptual expertise, such as forensic facial comparison (White, Phillips, Hahn, Hill, \& O'Toole, 2015), could inform theory and practice in ways that were previously not possible. More broadly, further investigation of whether these models adequately characterize the underlying dynamics of all kinds of decision processes may provide theoretically meaningful insights about the cognitive processes involved in decisions about naturalistic stimuli. 


\section{References}

Boag, R., Strickland, L., Heathcote, A., Neal, A., \& Loft, S. (2019). Cognitive Control and Capacity for Prospective Memory in Simulated Air Traffic Control. Journal of Experimental Psychology: General.

Brinberg D., \& McGrath J. E. (1985). Validity and the research process. Beverly Hills, CA: SAGE.

Brooks, S. P., \& Gelman, A. (1998). General methods for monitoring convergence of iterative simulations. Journal of Computational and Graphical Statistics, 7(4), 434-455.

Brown, S. D., \& Heathcote, A. (2008). The simplest complete model of choice response time: Linear ballistic accumulation. Cognitive Psychology, 57(3), 153-178. doi: 10.1016/j.cogpsych.2007.12.002

Bürkner, P.C. (2017). brms: An R Package for Bayesian Multilevel Models Using Stan. Journal of Statistical Software, 80(1), 1-28. doi:10.18637/jss.v080.i01

Donkin, C., Brown, S. D., \& Heathcote, A. (2009). The overconstraint of response time models: Rethinking the scaling problem. Psychonomic Bulletin \& Review, 16(6), 11291135. doi: 10.3758/PBR.16.6.1129

Donkin, C., Brown, S., Heathcote, A., \& Wagenmakers, E.-J. (2011). Diffusion versus linear ballistic accumulation: Different models but the same conclusions about psychological processes? Psychonomic Bulletin \& Review, 18, 61- 69. doi:10.3758/s13423-010-0022-4

Donkin, C., \& Brown, S. (2018). Response Times and Decision-making. In E. J. Wagenmakers (4th ed.) Stevens' Handbook of Experimental Psychology and Cognitive Neuroscience, Volume 5, Methodology (349-382). NY: Wiley.

Dror, I. E. (2018). Biases in forensic experts. Science, 6386(360), 243. doi: $10.1126 /$ science.aat 8443 
Green, D. M., \& Swets, J. A. (1966). Signal detection theory and psycho-physics. New York, NY: Wiley.

Heathcote, A., \& Love, J. (2012). Linear deterministic accumulator models of simple choice. Frontiers in Psychology, 3, 292. doi: 10.3389/fpsyg.2012.00292

Heathcote, A., Suraev, A., Curley, S., Love, J. \& Michie, P. (2015). Decision processes and the slowing of simple choices in schizophrenia. Journal of Abnormal Psychology, 124(4), 967-974, doi: /10.1037/abn0000117961

Heathcote, A ., Lin, Y. S., Reynolds, A., Strickland, L., Gretton, M. \& Matzke, D. (2018). Dynamic models of choice. Behavior Research Methods.

Ho, T. C., Yang, G., Wu, J., Cassey, P., Brown, S. D., Hoang, N., ... Yang, T. T. (2014) Functional connectivity of negative emotional processing in adolescent depression. Journal of Affective Disorders, 155, 65-74.

Hutchins, E. (1995). Cognition in the Wild. MIT Press.

Kruschke, J. K. (2014). Doing Bayesian data analysis: A tutorial with R, JAGS, and Stan. Academic Press: London.

Leite, F. P., \& Ratcliff, R. (2011). What cognitive processes drive response biases? A diffusion model analysis. Judgment and Decision Making, 6(7), 651-687.

Loft, S., Bolland, S., Humphreys, M., \& Neal, A. (2009). A theory and model of conflict detection in air traffic control: Incorporating environ- mental constraints. Journal of Experimental Psychology: Applied, 15, 106-124. doi: 10.1037/a0016118

Mook, D. G. (1983). In defense of external invalidity. Am Psychol, 38(4), 379-87. doi: 10.1037/0003-066X.38.4.379

Morrow, D. G., (2017). Publishing Papers That Matter. Journal of Experimental Psychology: Applied. doi: 10.1037/xap0000141 
Neal, A., \& Kwantes, P. J. (2009). An evidence accumulation model for conflict detection performance in a simulated air traffic control task. Human Factors, 51, 164-180. doi:10.1177/0018720809335071

Palada, H, Neal, A., Vuckovic, A., Martin, R., Samuels, K., \& Heathcote, A. (2016). Journal of Experimental Psychology: Applied, 22, 1-23. doi: http://dx.doi.org/10.1037/xap0000074

Palada, H., Neal, A., Tay, R., \& Heathcote, A. (2018). Understanding the causes of adapting, and failing to adapt, to time pressure in a complex multistimulus environment. Journal of Experimental Psychology: Applied, 24, 380-399. doi: 10.1037/xap0000176

Palada, H., Neal, A., Strayer, D., Ballard, T., \& Heathcote, A. (2019). Competing for cognitive resources: Measuring workload in a time pressured dual-task environment. Journal of Experimental Psychology: Human Perception \& Performance.

Rae, B., Heathcote, A., Donkin, C., Lee, A., \& Scott, B. (2014). The hare and the tortoise: Emphasizing speed can change the evidence used to make decisions. Journal of Experimental Psychology: Learning, Memory, \& Cognition, 40(5), 1226-1243. doi: $10.1037 / \mathrm{a} 0036801$

Ratcliff, R. (1978). A theory of memory retrieval. Psychological Review, 85(2), 59-108. https://doi.org/10.1037/0033-295X.85.2.59

Ratcliff, R., \& McKoon, G. (2008). The diffusion decision model: Theory and data for twochoice decision tasks. Neural Computation, 20(4), 873-922. doi:10.1162/neco.2008.12$06-420$

Ratcliff, R., \& Van Dongen, H. P. A. (2011). Diffusion model for one-choice reaction-time tasks and the cognitive effects of sleep deprivation. Proceedings of the National Academy of Sciences of the United States of America, 108(27), 11285-11290. 
Searston, R. A., Tangen, J. M., \& Eva, K. W. (2016). Putting bias into context: The role of familiarity in identification. Law and Human Behavior, 40 (1), 50-64. doi: $10.1037 / \mathrm{lhb} 0000154$

Searston, R. A., \& Tangen, J. M. (2017a). The style of a stranger: Identification expertise generalizes to coarser level categories. Psychonomic Bulletin \& Review, 24(4), 13241329. doi: 10.3758/s13423-016-1211-6

Searston, R. A., \& Tangen, J. M. (2017b). Expertise with unfamiliar objects is flexible to changes in task but not changes in class. PLoS ONE, 12(6): e0178403. doi: 10.1371/journal.pone.0178403

Searston, R. A., \& Tangen, J. M. (2017c). The emergence of perceptual expertise with fingerprints over time. Journal of Applied Research in Memory \& Cognition, 6(4), 442 451. doi: 10.1016/j.jarmac.2017.08.006

Strickland, L., Elliott, D., Wilson, M., Loft, S, Neal, A., \& Heathcote, A. (2019). Prospective memory in the red zone: Cognitive control and capacity sharing in a complex, multistimulus task. Journal of Experimental Psychology: Applied.

Tangen, J. M., Thompson, M. B., \& McCarthy, D. J. (2011). Identifying fingerprint expertise. Psychol. Sci. 22, 995-997. doi: 10.1177/09567976114 14729

Thompson, M. B., Tangen, J. M., \& McCarthy, D. J. (2013a). Expertise in fingerprint identification. Journal of Forensic Sciences, 58(6), 1519-1530. doi:10.1111/ 15564029.12203

Thompson, M. B., Tangen, J. M., and McCarthy, D. J. (2013b). Human matching performance of genuine crime scene latent fingerprints. Law Hum. Behav. 38, 84-93. doi: $10.1037 / \mathrm{hb} 0000051$

Thompson, M. B. \& Tangen, J. M. (2014). The nature of expertise in fingerprint matching: Experts can do a lot with a little. PLOS ONE, 9(12), e114759. doi: 10.1371/journal.pone.0114759 
Trueblood, J. S., Holmes, W. R., Seegmiller, A. C., Douds, J., Compton, M., Szentirmai, E., ... Eichbaum, Q. (2018). The impact of speed and bias on thecognitive processes of experts and novicesin medical image decision-making. Cognitive Research: Principles and Implications, 3(28). doi: 10.1186/s41235-018-0119-2

Turner, B. M., Sederberg, P. B., Brown, S. D., \& Steyvers, M. (2013). A method for efficiently sampling from distributions with correlated dimensions. Psychological Methods, 18(3), 368-384. doi: 10.1037/a0032222

Ulery, B. T., Hicklin, R. A., Buscaglia, J., \& Roberts, M. A. (2011). Accuracy and reliability of forensic latent fingerprint decisions. Proceedings of the National Academy of Sciences of the United States of America, 108(19), 7733-7738. doi:10.1073/Pnas.1018707108.

Vuckovic, A., Kwantes, P. J., Humphreys, M., \& Neal, A. (2014). A sequential sampling account of response bias and speed-accuracy tradeoffs in a conflict detection task. Journal of Experimental Psychology: Applied, 20, 55-68. http://dx.doi.org/10.1037/xap000007

White, D., Phillips, P. J., Hahn, C. A., Hill, M., \& O'Toole, A. J. (2015). Perceptual expertise in forensic facial image comparison. Proceedings of the Royal Society B: Biological Sciences, 282(1814). doi: 10.1098/rspb.2015.1292

Woods, D. D. (1985). The observation problem in psychology (Westinghouse Technical Report). Pittsburgh, PA: Westinghouse Corporation. 


\section{Supplementary Materials}

\section{Sampling Procedure}

We ran 33 chains, which is 3 times the number of individual-level parameters (the DMC package default). To reduce autocorrelation, we used a thinning interval on sampling procedure. For example, a thinning of 10 means that one sample was saved every 10 iterations.

For Experiment 1, we set a thinning interval of 10. Sampling was done by using automated routines enabled by the 'run.unstuck.dmc' and 'run.converge.dmc' functions, which sample until no chains are stuck and until the multivariate potential scale reduction factor is less than 1.1 for all participants (Gelman \& Brooks, 1998). After convergence, we discarded all previous samples and sampled for a further 1500 iterations. In this analysis, there was some non-stationarity in the chains even after the automated convergence routine had been completed. We therefore discarded the first 1000 of the 1500 final samples in each chain, which removed this non-stationarity. Visual inspection of the final 500 samples confirmed that the chains were well mixed and stationary.

For Experiment 2, we initially set a thinning interval of 15, though subsequently increased this to 25 to better reduce autocorrelation. We first ran the 'run.unstuck.dmc' procedure until chains were unstuck. Thereafter, rather than using auto-convergence routines we used the 'h.run.dmc' procedure to run hierarchical sampling iterations. We initially ran the procedure to collect 1400 iterations. Visual inspections of the final 500 iterations confirmed that the chains were well mixed and stationary. From there, we collected a further 300 samples with a thinning set to 10; this last step was also run to ensure that the chains did not expand, as this behavior was observed in prior attempts to fit the model. For Experiment 3, we set a thinning of 25. We used the procedure as in Experiment 2 to ensure that chains were unstuck. Thereafter, we initially collected 1100 iterations and then collected a final 500 iterations for analysis. 


\section{Priors}

The population-level parameters included two parameters: Location and scale. The prior distributions and values for the location parameter is shown in Table 1. After having fit Experiment 1, we reviewed the estimates and found that the estimated mean of the start-point (A) was 7.16, which is far from the initial prior specified. Therefore, in Experiments 2 and 3 we updated the location of the start-point to 5.0. All scale parameters were modeling using a zero-truncated normal distribution with a mean of 0 and a standard deviation of 1 .

Table 1.

Priors for location parameter of the population level LBA model parameters

\begin{tabular}{lccccc}
\hline Model Parameter & Distribution & Mean & Standard Deviation & Lower & Upper \\
\hline $\begin{array}{l}\text { Start-point } \\
(A)\end{array}$ & Truncated normal & $1.00 / 5.00$ & 1.00 & 0 & NA \\
$\begin{array}{l}\text { Threshold } \\
(B)\end{array}$ & Truncated normal & 1.00 & 1.00 & 0 & NA \\
$\begin{array}{l}\text { Rate } \\
(v)\end{array}$ & Normal & 1.00 & 2.00 & NA & NA \\
$\begin{array}{l}\text { Rate Variability } \\
(s v)\end{array}$ & Truncated normal & 1.00 & 1.00 & 0 & NA \\
$\begin{array}{l}\text { Non-decision } \\
(t e r)\end{array}$ & Truncated normal & 0.30 & 1.00 & 0.10 & 1.00 \\
\hline
\end{tabular}

